\title{
Asymptotic behaviour of a cylindrical elastic structure periodically reinforced along identical fibers
}

\author{
Mustapha EL JARROUDI* \\ Département de Mathématiques, Faculté des Sciences et Techniques \\ B.P. 416, Tanger, MOROCCO \\ and \\ Alain BRILLARD ${ }^{\dagger}$ \\ Université de Haute-Alsace, 2 rue des Frères Lumière, \\ F-68093 Mulhouse Cedex, FRANCE
}

\begin{abstract}
We describe the asymptotic behaviour of a cylindrical elastic body, reinforced along identical $\varepsilon$-periodically distributed fibers of size $r_{\varepsilon}$, with $0<r_{\varepsilon}<\varepsilon$, filled in with some different elastic material, when this small parameter $\varepsilon$ goes to 0 . The case of small deformations and small strains is considered. We exhibit a critical size of the fibers and a critical link between the radius of the fibers and the size of the Lamé coefficients of the reinforcing elastic material. Epi-convergence arguments are used in order to prove this asymptotic behaviour. The proof is essentially based on the construction of appropriate test-functions.
\end{abstract}

Keywords : Reinforcement, fibers, linear elasticity, epi-convergence.

AMS Subject Classification (2000) : 74E30, 74G10, 35B27

\section{Introduction}

The purpose of this work is to determine the asymptotic behaviour of an elastic material periodically reinforced by means of identical fibers filled in with some isotropic and homogeneous elastic material. In the first part, the fibers are longitudinally distributed inside the elastic material. The limit law is derived, studying the convergence of the elastic energy, and we exhibit a critical size of the fibers and a critical size of the Lamé coefficients

\footnotetext{
*Email: eljar@fstt.ac.ma

${ }^{\dagger}$ Corresponding author. Email : A.Brillard@uha.fr
} 
of the reinforcing fibers. In the last part of this work, we suppose that the fibers are transversally distributed and we exhibit the limit law, which still involves a critical size and a critical size of the Lamé coefficients of the fibers, but working in a different limit functional space. These configurations intend to modelize, for example, the behaviour of a strap reinforced by means of identical fibers which are longitudinally or transversally disposed inside the strap.

Let $\omega$ be a bounded, smooth and open subset of $\mathbf{R}^{2}$ and $\left.\Omega=\omega \times\right] 0, L\left[\subset \mathbf{R}^{3}\right.$, where $L$ is positive. $\Gamma_{1}$ denotes the lower basis of $\Omega: \Gamma_{1}=\omega \times\{0\}, \Gamma_{2}$ its upper basis : $\Gamma_{2}=\omega \times\{L\}$ and $\Sigma$ its lateral surface : $\left.\Sigma=\partial \omega \times\right] 0, L[$.

Let $\varepsilon$ be some positive real. In the first part of this work, we dispose inside $\Omega$ longitudinal fibers. More precisely, for every $k=\left(k_{1}, k_{2}\right)$ in $\mathbf{Z}^{2}$, we define the square : $\left.Y_{\varepsilon}^{k}=\left(\varepsilon k_{1}, \varepsilon k_{2}\right)+\right]-\varepsilon / 2, \varepsilon / 2\left[^{2}\right.$. Then we denote by $Y_{\varepsilon}$ the union of all the $\varepsilon$-cells $Y_{\varepsilon}^{k}$ included in $\omega: Y_{\varepsilon}=\cup_{k \in K(\varepsilon)} Y_{\varepsilon}^{k}$. Choosing a parameter $r_{\varepsilon}$ smaller than $\varepsilon$, we consider the disk $D_{\varepsilon}^{k}$ of radius $r_{\varepsilon}$ contained in $Y_{\varepsilon}^{k}$ and the cylinder $\left.T_{\varepsilon}^{k}=D_{\varepsilon}^{k} \times\right] 0, L\left[. T_{\varepsilon}\right.$ denotes the union $\cup_{k} T_{\varepsilon}^{k}$ of the cylinders $T_{\varepsilon}^{k}$ contained in $\Omega$. Thus $\overline{T_{\varepsilon}} \cap \Sigma$ is empty. The total number of such cylinders contained in $\Omega$ (that is the cardinal of $K(\varepsilon)$ ) is equivalent to $|\omega| / \varepsilon^{2}$, with $|\omega|=\operatorname{area}(\omega)$. The domain $\Omega_{\varepsilon}=\Omega \backslash \overline{T_{\varepsilon}}$ is supposed to be the reference configuration of some linear elastic, homogeneous and isotropic material, thus satisfying the following Hooke's law

$$
\sigma_{i j}(u)=\lambda e_{m m}(u) \delta_{i j}+2 \mu e_{i j}(u), \quad i, j, m=1,2,3,
$$

where the summation convention has been used with respect to repeated indices, $\lambda$ and $\mu$ are the Lamé coefficients of the material, satisfying : $\mu>0$ and $\lambda \geq 0, \delta_{i j}$ is Kronecker's symbol and $e(u)$ is the linearized deformation tensor, the components of which are given by : $e_{i j}(u)=\frac{1}{2}\left(\frac{\partial u_{j}}{\partial x_{i}}+\frac{\partial u_{i}}{\partial x_{j}}\right)$.

Figure 1: The domain $\Omega$ and the cylinders $T_{\varepsilon}^{k}$.

We suppose that $T_{\varepsilon}$ is the reference configuration of some linear elastic, homogeneous and isotropic material satisfying Hooke's law

$$
\sigma_{i j}^{\varepsilon}(u)=\lambda^{\varepsilon} e_{m m}(u) \delta_{i j}+2 \mu^{\varepsilon} e_{i j}(u), \quad i, j, m=1,2,3,
$$


where the Lamé coefficients $\lambda^{\varepsilon} \geq 0$ and $\mu^{\varepsilon}>0$ depend on $\varepsilon$ and satisfy

$$
\exists c>0, \forall \varepsilon>0: \mu^{\varepsilon} \geq c .
$$

The structure $\Omega$ built with these two elastic materials is submitted to some volumic forces the density of which $f=\left(f_{1}, f_{2}, f_{3}\right)$ belongs to $L^{2}\left(\Omega, \mathbf{R}^{3}\right)$. We suppose that the structure is held fixed along $\Gamma_{1}$ and that the tractions are equal to 0 on the rest of the boundary : $\sigma_{i j}\left(u^{\varepsilon}\right) n_{j}=0, i, j=1,2,3$, where $n$ is the unit outer normal to the boundary. Let us introduce the functional $F^{\varepsilon}$ defined on $H^{1}\left(\Omega, \mathbf{R}^{3}\right)$ by:

$$
F^{\varepsilon}(u)= \begin{cases}\int_{\Omega_{\varepsilon}} \sigma_{i j}(u) e_{i j}(u) d x+\int_{T_{\varepsilon}} \sigma_{i j}^{\varepsilon}(u) e_{i j}(u) d x & \text { if } u \in H_{\Gamma_{1}}^{1}\left(\Omega, \mathbf{R}^{3}\right) \\ +\infty & \text { otherwise, }\end{cases}
$$

with : $H_{\Gamma_{1}}^{1}\left(\Omega, \mathbf{R}^{3}\right)=\left\{u \in H^{1}\left(\Omega, \mathbf{R}^{3}\right) \mid u=0\right.$ on $\left.\Gamma_{1}\right\}$. The problem under consideration can be associated to the minimization problem involving the functional $F^{\varepsilon}$, as indicated in the following

Lemma 1 1. The minimization problem:

$$
\min _{u \in H^{1}\left(\Omega, \mathbf{R}^{3}\right)}\left\{F^{\varepsilon}(u)-2 \int_{\Omega} f . u d x\right\}
$$

admits a unique solution $u^{\varepsilon}$ belonging to $H_{\Gamma_{1}}^{1}\left(\Omega, \mathbf{R}^{3}\right)$ and which satisfies the variational formulation:

$$
\int_{\Omega_{\varepsilon}} \sigma_{i j}\left(u^{\varepsilon}\right) e_{i j}(u) d x+\int_{T_{\varepsilon}} \sigma_{i j}^{\varepsilon}\left(u^{\varepsilon}\right) e_{i j}(u) d x=\int_{\Omega} f . u d x, \quad \forall u \in H_{\Gamma_{1}}^{1}\left(\Omega, \mathbf{R}^{3}\right)
$$

and is a weak solution of the problem:

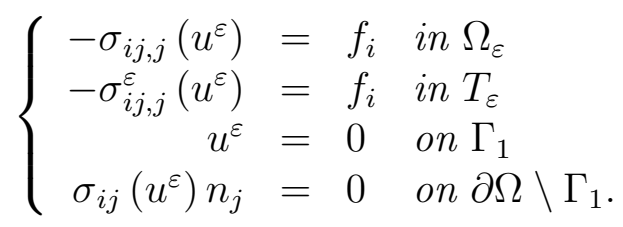

2. The sequence $\left(u^{\varepsilon}\right)_{\varepsilon}$ is bounded in $H^{1}\left(\Omega, \mathbf{R}^{3}\right)$.

3. Assume that $: \sup _{\varepsilon}\left(-\varepsilon^{2} \ln \left(r_{\varepsilon}\right)\right)<+\infty$. Then, $\sup _{\varepsilon}\left(\left(\int_{T_{\varepsilon}}\left|u^{\varepsilon}\right|^{2} d x\right) /\left|T_{\varepsilon}\right|\right)$ is finite and if $R^{\varepsilon}\left(u^{\varepsilon}\right)$ is the rescaled restriction of $u^{\varepsilon}$ to the fibers defined by:

$$
R^{\varepsilon}\left(u^{\varepsilon}\right)=\frac{|\Omega|}{\left|T_{\varepsilon}\right|} u^{\varepsilon} \mathbf{1}_{T_{\varepsilon}}
$$

where $|\Omega|$ means the volume of $\Omega$ and $\mathbf{1}_{T_{\varepsilon}}$ denotes the characteristic function of $T_{\varepsilon}$, the sequence $\left(R^{\varepsilon}\left(u^{\varepsilon}\right)\right)_{\varepsilon}$ is bounded in $L^{1}\left(\mathbf{R}^{3}, \mathbf{R}^{3}\right)$. 
Proof. 1. Because $\lambda^{\varepsilon}$ is nonnegative, we write for every $u$ in $H_{\Gamma_{1}}^{1}\left(\Omega, \mathbf{R}^{3}\right)$

$$
F^{\varepsilon}(u) \geq \inf \left(2 \mu, 2 \mu^{\varepsilon}\right) \int_{\Omega} e_{i j}(u) e_{i j}(u) d x \geq C \inf \left(2 \mu, 2 \mu^{\varepsilon}\right) \int_{\Omega}|\nabla u|^{2} d x,
$$

using the classical Korn's inequality, because $u$ vanishes on $\Gamma_{1}$. The hypothesis (3) and this inequality imply that $F^{\varepsilon}$ is coercive on $H^{1}\left(\Omega, \mathbf{R}^{3}\right)$. Moreover, $F^{\varepsilon}$ is lower semi-continuous for the weak topology of $H_{\Gamma_{1}}^{1}\left(\Omega, \mathbf{R}^{3}\right)$ and is not identically equal to $+\infty$. Thus, classical convex analysis results imply the existence and the uniqueness of a minimizer $u^{\varepsilon}$ of $F^{\varepsilon}$ on $H_{\Gamma_{1}}^{1}\left(\Omega, \mathbf{R}^{3}\right)$, which satisfies the variational formulation (6) and, thus, is a weak solution of (7).

2. We observe that: $F^{\varepsilon}\left(u^{\varepsilon}\right)-2 \int_{\Omega} f \cdot u^{\varepsilon} d x \leq F^{\varepsilon}(0)=0$, which implies, using the preceding inequality, that

$$
C \inf \left(2 \mu, 2 \mu^{\varepsilon}\right) \int_{\Omega}\left|\nabla u^{\varepsilon}\right|^{2} d x \leq 2\|f\|_{L^{2}(\Omega)}\left\|u^{\varepsilon}\right\|_{L^{2}(\Omega)} .
$$

Using Poincaré's inequality, we thus deduce that $\left(u^{\varepsilon}\right)_{\varepsilon}$ is bounded in $H_{\Gamma_{1}}^{1}\left(\Omega, \mathbf{R}^{3}\right)$. 3. Before proving this assertion, let us first recall the following estimate, which has been proved in [6]

Lemma 2 There exists some positive constant $C$ such that, for every u in $H^{1}\left(\Omega, \mathbf{R}^{3}\right)$, one has:

$$
\frac{1}{\left|T_{\varepsilon}\right|} \int_{T_{\varepsilon}} u^{2} d x \leq C\left(\int_{\Omega}|\nabla u|^{2} d x-\varepsilon^{2} \ln \left(r_{\varepsilon}\right)+\varepsilon^{2}\right) .
$$

Proof. We first define : $u^{\prime}(r, \theta, z):=u\left(\varepsilon k_{1}+r \cos (\theta), \varepsilon k_{2}+r \cos (\theta), z\right)$, in the fiber centred at $\left(\varepsilon k_{1}, \varepsilon k_{2}\right)$. Then, we observe that, for every $r_{1} \leq r_{2}<\varepsilon / 2$

$$
\begin{gathered}
u^{\prime}\left(r_{2}, \theta, z\right)-u^{\prime}\left(r_{1}, \theta, z\right)=\left(r_{2}-r_{1}\right) \int_{0}^{1} \frac{\partial u^{\prime}}{\partial r}\left((1-t) r_{1}+t r_{2}\right) \frac{\sqrt{(1-t) r_{1}+t r_{2}}}{\sqrt{(1-t) r_{1}+t r_{2}}} d t \\
\Rightarrow\left(u^{\prime}\left(r_{2}, \theta, z\right)-u^{\prime}\left(r_{1}, \theta, z\right)\right)^{2} \leq\left(\ln \left(r_{2}\right)-\ln \left(r_{1}\right)\right) \int_{r_{1}}^{r_{2}}\left(\frac{\partial u^{\prime}}{\partial r}\right)^{2} r d r .
\end{gathered}
$$

Defining : $f(r)=\sum_{k \in K(\varepsilon)} \int_{0}^{L} \int_{0}^{2 \pi}\left(u^{\prime}\right)^{2}(r, \theta, z) d \theta d z$, the previous inequality implies : $f\left(r_{1}\right) \leq 2 f\left(r_{2}\right)+2\|\nabla u\|_{L^{2}\left(\Omega, \mathbf{R}^{3}\right)}^{2} \ln \left(r_{2} / r_{1}\right)$, which implies, for every $r_{2}$ in $[\varepsilon / 4, \varepsilon / 2]$

$$
\begin{aligned}
\frac{1}{\left|T_{\varepsilon}\right|} \int_{T_{\varepsilon}} u^{2} d x & =\frac{1}{\left|T_{\varepsilon}\right|} \int_{0}^{r_{\varepsilon}} f(r) r d r \\
& \leq \frac{2}{\left|T_{\varepsilon}\right|} \int_{0}^{r_{\varepsilon}}\left(f\left(r_{2}\right)+\|\nabla u\|_{L^{2}\left(\Omega, \mathbf{R}^{3}\right)}^{2}\left(\ln \left(r_{2}\right)-\ln (r)\right)\right) r d r \\
& \leq \frac{C \varepsilon^{2}}{\left(r_{\varepsilon}\right)^{2}}\left(f\left(r_{2}\right)\left(r_{\varepsilon}\right)^{2}+\|\nabla u\|_{L^{2}\left(\Omega, \mathbf{R}^{3}\right)}^{2}\left(\left(r_{\varepsilon}\right)^{2}-\frac{\left(r_{\varepsilon}\right)^{2}}{2} \ln \left(r_{\varepsilon}\right)+\frac{\left(r_{\varepsilon}\right)^{2}}{4}\right)\right) \\
& \leq C\left(f\left(r_{2}\right) \varepsilon^{2}+\|\nabla u\|_{L^{2}\left(\Omega, \mathbf{R}^{3}\right)}^{2} \varepsilon^{2}-\frac{\varepsilon^{2}}{2} \ln \left(r_{\varepsilon}\right)+\frac{\varepsilon^{2}}{4}\right) \\
& \leq C\left(4 f\left(r_{2}\right) \varepsilon r_{2}+\|\nabla u\|_{L^{2}\left(\Omega, \mathbf{R}^{3}\right)}^{2} \varepsilon^{2}-\frac{\varepsilon^{2}}{2} \ln \left(r_{\varepsilon}\right)+\frac{\varepsilon^{2}}{4}\right)
\end{aligned}
$$


and then, taking the mean value of this inequality with respect to $r_{2}$ in $[\varepsilon / 4, \varepsilon / 2]$

$$
\begin{aligned}
\frac{1}{\left|T_{\varepsilon}\right|} \int_{T_{\varepsilon}} u^{2} d x & \leq C\left(16 \int_{\varepsilon / 4}^{\varepsilon / 2} f(r) r d r+\|\nabla u\|_{L^{2}\left(\Omega, \mathbf{R}^{3}\right)}^{2} \varepsilon^{2}-\frac{\varepsilon^{2}}{2} \ln \left(r_{\varepsilon}\right)+\frac{\varepsilon^{2}}{4}\right) \\
& \leq C\left(\left(16+\varepsilon^{2}\right)\|\nabla u\|_{L^{2}\left(\Omega, \mathbf{R}^{3}\right)}^{2}-\frac{\varepsilon^{2}}{2} \ln \left(r_{\varepsilon}\right)+\frac{\varepsilon^{2}}{4}\right) .
\end{aligned}
$$

Coming back to the proof of Lemma 1, we observe that Lemma 2 implies that $\sup _{\varepsilon}\left(\left(\int_{T_{\varepsilon}}\left|u^{\varepsilon}\right|^{2} d x\right) /\left|T_{\varepsilon}\right|\right)$ is finite, as soon as $\sup _{\varepsilon}\left(-\varepsilon^{2} \ln \left(r_{\varepsilon}\right)\right)<+\infty$. Then, using Cauchy-Schwarz inequality, we finally prove that the quantity $\left(\int_{\mathbf{R}^{3}}\left|R^{\varepsilon}\left(u^{\varepsilon}\right)\right| d x\right)_{\varepsilon}$ is bounded, which ends the proof of Lemma 1 .

In the sequel, we will assume that the hypothesis $\sup _{\varepsilon}\left(-\varepsilon^{2} \ln \left(r_{\varepsilon}\right)\right)<+\infty$ is always satisfied.

Our purpose is to describe the asymptotic behaviour of $\left(u^{\varepsilon}\right)_{\varepsilon}$ and that of $\left(R^{\varepsilon}\left(u^{\varepsilon}\right)\right)_{\varepsilon}$, when $\varepsilon$ goes to 0 . This will be obtained using epi-convergence arguments, that is studying the asymptotic behaviour of the sequence $\left(F^{\varepsilon}\right)_{\varepsilon}$, when $\varepsilon$ goes to 0 . We will first suppose that the coefficients $\lambda_{o}$ and $\mu_{o}$, defined by

$$
\lambda_{o}=\lim _{\varepsilon \rightarrow 0} \frac{\lambda^{\varepsilon}\left(r_{\varepsilon}\right)^{2}}{\varepsilon^{2}}, \mu_{o}=\lim _{\varepsilon \rightarrow 0} \frac{\mu^{\varepsilon}\left(r_{\varepsilon}\right)^{2}}{\varepsilon^{2}} .
$$

are finite and $\mu_{o}$ is positive. Thanks to the properties of the epi-convergence, we then derive the asymptotic behaviour of the solution in many other cases.

This kind of reinforcement problems follows earlier works like [2], 3], [6], for example. However, the works [2] and [3] were dealing with scalar problems (also involving the $p$ laplacian operator). The work [6] is dealing with linear elasticity problems but assuming another scaling of the coefficients, which will be described later on in the present work. The work [4] deals with the homogenization of composite media evoking the vectorial case. See also [5] for similar phenomena in a quite general situation.

\section{Construction and study of the test-functions}

We define

$$
\begin{aligned}
D & =\left\{\left(y_{1}, y_{2}\right) \in \mathbf{R}^{2} \mid\left(y_{1}\right)^{2}+\left(y_{2}\right)^{2}<1\right\} \\
D\left(r, r^{\prime}\right) & =\left\{\left(y_{1}, y_{2}\right) \in \mathbf{R}^{2} \mid r^{2}<\left(y_{1}\right)^{2}+\left(y_{2}\right)^{2}<r^{2}\right\} \\
S_{r} & =\left\{\left(y_{1}, y_{2}\right) \in \mathbf{R}^{2} \mid\left(y_{1}\right)^{2}+\left(y_{2}\right)^{2}=r^{2}\right\}
\end{aligned}
$$

for $0<r<r^{\prime}$, and for every $k=\left(k_{1}, k_{2}\right)$ in $\mathbf{Z}^{2}$

$$
\begin{aligned}
& B_{\varepsilon}^{k}=\left\{\left(x_{1}, x_{2}, x_{3}\right) \mid\left(x_{1}-k_{1} \varepsilon\right)^{2}+\left(x_{2}-k_{2} \varepsilon\right)^{2}<\left(s_{\varepsilon}\right)^{2}, x_{3} \in\right] 0, L[\} \\
& C_{\varepsilon}^{k}=\left\{\left(x_{1}, x_{2}, x_{3}\right) \mid\left(r_{\varepsilon}\right)^{2}<\left(x_{1}-k_{1} \varepsilon\right)^{2}+\left(x_{2}-k_{2} \varepsilon\right)^{2}<\left(s_{\varepsilon}\right)^{2}, x_{3} \in\right] 0, L[\},
\end{aligned}
$$

choosing $s_{\varepsilon}$ such that 


$$
\lim _{\varepsilon \rightarrow 0} \frac{s_{\varepsilon}}{\varepsilon}=0=\lim _{\varepsilon \rightarrow 0} \frac{r_{\varepsilon}}{s_{\varepsilon}}=0=\lim _{\varepsilon \rightarrow 0} \varepsilon^{2} \ln ^{2} s_{\varepsilon} .
$$

Finally, we denote: $B_{\varepsilon}=\cup_{k} B_{\varepsilon}^{k}, C_{\varepsilon}=\cup_{k} C_{\varepsilon}^{k}$.

We introduce the solution $w^{m}=\left(w_{1}^{m}, w_{2}^{m}\right), m=1,2$, of the linear plane elasticity problems

$$
\left\{\begin{aligned}
\sigma_{i j, j}\left(w^{m}\right)(y) & =0 & & \forall y \in \mathbf{R}^{2} \backslash \bar{D}, i, j=1,2 \\
w^{m}(y) & =0 & & \text { on } S_{1} \\
w_{m}^{m}(y) & \simeq-\ln |y|+C t e & & \text { when }|y| \rightarrow \infty \\
\left|w_{p}^{m}\right|(y) & \leq C t e & & \text { when }\left\{\begin{array}{l}
p=2 \text { if } m=1 \\
p=1 \text { if } m=2
\end{array}\right.
\end{aligned}\right.
$$

where: $\sigma_{i j}\left(w^{m}\right)=\lambda e_{i j}\left(w^{m}\right)+2 \mu e_{i j}\left(w^{m}\right)$. Thanks to the potential theory methods, described for example in [7], the solution $w^{m}$ of (11) can be computed as

$$
\left\{\begin{array}{l}
w_{1}^{1}\left(y_{1}, y_{2}\right)=-\ln |y|+\frac{\left(y_{2}\right)^{2}-\left(y_{1}\right)^{2}}{2 \kappa|y|^{2}}-\frac{\left(y_{2}\right)^{2}-\left(y_{1}\right)^{2}}{2 \kappa|y|^{4}} \\
w_{2}^{1}\left(y_{1}, y_{2}\right)=\frac{y_{2} y_{1}}{\kappa|y|^{2}}-\frac{y_{2} y_{1}}{\kappa|y|^{4}} \\
w_{1}^{2}\left(y_{1}, y_{2}\right)=\frac{y_{2} y_{1}}{\kappa|y|^{2}}-\frac{y_{2} y_{1}}{|y|^{4}} \\
w_{2}^{2}\left(y_{1}, y_{2}\right)=-\ln |y|-\frac{\left(y_{2}\right)^{2}-\left(y_{1}\right)^{2}}{2 \kappa|y|^{2}}+\frac{\left(y_{2}\right)^{2}-\left(y_{1}\right)^{2}}{2 \kappa|y|^{4}}
\end{array}\right.
$$

with : $\kappa=(\lambda+3 \mu) /(\lambda+\mu)$. We also introduce the function $w\left(y_{1}, y_{2}\right)=-\ln |y|$, which is harmonic in $\mathbf{R}^{2} \backslash\{0\}$ and verifies the following properties

$$
w_{\mid S_{1}}=0, \lim _{|y| \rightarrow \infty} \frac{w\left(y_{1}, y_{2}\right)}{\ln |y|}=-1, \int_{S_{1}} \frac{\partial w}{\partial n} d \sigma=2 \pi .
$$

Let us observe that

Lemma 3 One has the following convergences:

$$
\begin{aligned}
& \text { 1. } \lim _{R \rightarrow+\infty} \frac{1}{\ln R} \int_{D(1, R)} \sigma_{i j}\left(w^{m}\right) e_{i j}\left(w^{l}\right) d y=\frac{2 \pi \mu(1+\kappa)}{\kappa} \delta_{l m} . \\
& \text { 2. } \lim _{R \rightarrow+\infty} \frac{1}{\ln R} \int_{D(1, R)}|\nabla w|^{2} d y=2 \pi,
\end{aligned}
$$

Proof. The proof is trivial.

Using the solutions of these plane problems, we now build the functions $w_{\varepsilon}^{m k}$, for every $k=\left(k_{1}, k_{2}\right)$ as 


$$
\begin{aligned}
& w_{\varepsilon}^{\alpha k}\left(x_{1}, x_{2}\right)=\frac{-1}{\ln r_{\varepsilon}}\left(\begin{array}{c}
w_{1}^{\alpha}\left(\frac{x_{1}-k_{1} \varepsilon}{r_{\varepsilon}}, \frac{x_{2}-k_{2} \varepsilon}{r_{\varepsilon}}\right) \\
w_{2}^{\alpha}\left(\frac{x_{1}-k_{1} \varepsilon}{r_{\varepsilon}}, \frac{x_{2}-k_{2} \varepsilon}{r_{\varepsilon}}\right) \\
0
\end{array}\right) \\
& w_{\varepsilon}^{3 k}\left(x_{1}, x_{2}\right)=\frac{-1}{\ln r_{\varepsilon}}\left(w\left(\frac{x_{1}-k_{1} \varepsilon}{r_{\varepsilon}}, \frac{x_{2}-k_{2} \varepsilon}{r_{\varepsilon}}\right)\right),
\end{aligned}
$$

$\alpha=1,2$. These functions $w_{\varepsilon}^{m k}$ satisfy the following properties.

Lemma 4 There exist two positive constants $C_{0}$ and $C_{1}$, independant of $\varepsilon$, such that:

1. $\left|e_{m}-w_{\varepsilon}^{m k}\right|^{2} \leq C_{0} \frac{\ln ^{2}\left(R_{\varepsilon}^{k}\right)+1}{\ln ^{2}\left(r_{\varepsilon}\right)}$, in $B_{\varepsilon}^{k}$,

2. $\left|\frac{\partial w_{\varepsilon}^{m k}}{\partial x_{i}}\right|^{2} \leq \frac{C_{1}}{\left(R_{\varepsilon}^{k}\right)^{2} \ln ^{2}\left(r_{\varepsilon}\right)}$, in $B_{\varepsilon}^{k}, i=1,2,3$,

where $e_{m}$ is the $m$-th vector of the canonical basis of $\mathbf{R}^{3}$ and

$$
\left(R_{\varepsilon}^{k}\right)^{2}=\left(x_{1}-k_{1} \varepsilon\right)^{2}+\left(x_{2}-k_{2} \varepsilon\right)^{2} .
$$

Proof. Immediate, thanks to the expression of $w_{\varepsilon}^{m k}$.

Lemma 5 If $\gamma:=\lim _{\varepsilon \rightarrow 0}\left(-1 /\left(\varepsilon^{2} \ln r_{\varepsilon}\right)\right)$ is finite, then:

1. For every $m$ and $l$, one has

$$
\lim _{\varepsilon \rightarrow 0} \int_{B_{\varepsilon}} \sigma_{i j}\left(w_{\varepsilon}^{m k}\right) e_{i j}\left(w_{\varepsilon}^{l k}\right) d x= \begin{cases}\frac{2 \pi \gamma \mu(1+\kappa)}{\kappa}|\Omega| \delta_{l m} & m, l=1,2 \\ 0 & l=3, m=1,2 \\ 2 \pi \gamma \mu|\Omega| & m, l=3 .\end{cases}
$$

2. Let $\varphi$ be any element of $C^{1}(\bar{\Omega})$. Then

$$
\lim _{\varepsilon \rightarrow 0} \int_{B_{\varepsilon}} \sigma_{i j}\left(w_{\varepsilon}^{m k}\right) e_{i j}\left(w_{\varepsilon}^{l k}\right) \varphi d x= \begin{cases}\frac{2 \pi \gamma \mu(1+\kappa)}{\kappa} \delta_{l m} \int_{\Omega} \varphi d x & m, l=1,2 \\ 0 & l=3, m=1,2 \\ 2 \pi \gamma \mu \int_{\Omega} \varphi d x & m, l=3 .\end{cases}
$$


3. Let $\varphi_{\varepsilon}^{k}$ be the truncation function defined by

$$
\varphi_{\varepsilon}^{k}(x)=\varphi_{\varepsilon}^{k}\left(x_{1}, x_{2}\right)= \begin{cases}\frac{-4}{3\left(s_{\varepsilon}\right)^{2}}\left(\left(R_{\varepsilon}^{k}\right)^{2}-\left(s_{\varepsilon}\right)^{2}\right) & \text { if } \frac{s_{\varepsilon}}{2} \leq R_{\varepsilon}^{k} \leq s_{\varepsilon} \\ 1 & \text { if } R_{\varepsilon}^{k} \leq \frac{s_{\varepsilon}}{2} \\ 0 & \text { if } R_{\varepsilon}^{k} \geq s_{\varepsilon}\end{cases}
$$

and $z_{\varepsilon}^{m}$ the function defined by

$$
z_{\varepsilon}^{m}(x)= \begin{cases}\varphi_{\varepsilon}^{k}(x)\left(e_{m}-w_{\varepsilon}^{m k}\right)(x) & \forall x \in B_{\varepsilon}^{k}, \forall k \\ 0 & \forall x \in \Omega \backslash \overline{B_{\varepsilon}}\end{cases}
$$

Then $\left(z_{\varepsilon}^{m}\right)_{\mid T_{\varepsilon}}=e_{m},\left(z_{\varepsilon}^{m}\right)_{\varepsilon}$ converges to 0 in the weak topology of $H^{1}\left(\Omega, \mathbf{R}^{3}\right)$ and

$$
\lim _{\varepsilon \rightarrow 0} \int_{\Omega} \sigma_{i j}\left(z_{\varepsilon}^{m}\right) e_{i j}\left(z_{\varepsilon}^{l}\right) d x= \begin{cases}\frac{2 \pi \gamma \mu(1+\kappa)}{\kappa}|\Omega| \delta_{l m} & \text { if } m, l=1,2 \\ 0 & \text { if } l=3, m=1,2 \\ 2 \pi \gamma \mu|\Omega| & \text { if } m, l=3 .\end{cases}
$$

Proof. 1. Using Hooke's law, the above expression of $w_{\varepsilon}^{m k}$ and the estimates given in Lemma 4, one has, for $m, l=1,3$

$$
\lim _{\varepsilon \rightarrow 0} \int_{C_{\varepsilon}} \sigma_{i j}\left(w_{\varepsilon}^{m k}\right) e_{i j}\left(w_{\varepsilon}^{l k}\right) d x=\frac{|\Omega|}{\varepsilon^{2} \ln ^{2} r_{\varepsilon}} \int_{D\left(1, s_{\varepsilon} / r_{\varepsilon}\right)} \sigma_{i j}\left(w^{m}\right) e_{i j}\left(w^{l}\right) d y_{1} d y_{2}+o_{\varepsilon}
$$

where: $y_{1}=\left(x_{1}-k_{1} \varepsilon\right) / r_{\varepsilon}, y_{2}=\left(x_{2}-k \varepsilon\right) / r_{\varepsilon}, \sigma_{i j}$ and $e_{i j}$ respectively denote the stress and the deformation tensors in the plane, with the Lamé coefficients $\lambda$ and $\mu$ and $\lim _{\varepsilon \rightarrow 0} o_{\varepsilon}=0$. One deduces from Lemma 3, through the definition of $s_{\varepsilon}$ that

$$
\lim _{\varepsilon \rightarrow 0} \frac{-1}{\ln r_{\varepsilon}} \int_{D\left(1, s_{\varepsilon} / r_{\varepsilon}\right)} \sigma_{i j}\left(w^{m}\right) e_{i j}\left(w^{l}\right) d y_{1} d y_{2}=\frac{2 \pi \mu(1+\kappa)}{\kappa} \delta_{m l},
$$

the other cases being treated in a similar way. We conclude, using the definition of $\gamma$. 2. The smoothness of $\varphi$ implies that for every $\left(x_{1}, x_{2}, x_{3}\right)$ in $C_{\varepsilon}^{k}$ we have : $\varphi\left(x_{1}, x_{2}, x_{3}\right)=$ $\varphi\left(k_{1} \varepsilon, k_{2} \varepsilon, x_{3}\right)+O\left(R_{\varepsilon}^{k}\right)$, which implies

$$
\begin{aligned}
& \int_{C_{\varepsilon}} \sigma_{i j}\left(w_{\varepsilon}^{m k}\right) e_{i j}\left(w_{\varepsilon}^{l k}\right) \varphi d x \\
& =\frac{1}{\varepsilon^{2} \ln ^{2} r_{\varepsilon}}\left(\int_{D\left(1, s_{\varepsilon} / r_{\varepsilon}\right)} \sigma_{i j}\left(w^{m}\right) e_{i j}\left(w^{l}\right) d y_{1} d y_{2}\left(\sum_{k} \varepsilon^{2} \int_{0}^{L} \varphi\left(k_{1} \varepsilon, k_{2} \varepsilon, x_{3}\right) d x_{3}\right)\right)+o_{\varepsilon} .
\end{aligned}
$$

But the smoothness of $\varphi$ also implies

$$
\lim _{\varepsilon \rightarrow 0} \sum_{k} \varepsilon^{2} \int_{0}^{L} \varphi\left(k_{1} \varepsilon, k_{2} \varepsilon, x_{3}\right) d x_{3}=\int_{\Omega} \varphi d x
$$


from which we conclude, using the first assertion.

3. We observe that $\varphi_{\varepsilon}^{k} \equiv 0$ in $\Omega \backslash \overline{B_{\varepsilon}}$ and $w_{\varepsilon}^{m k} \equiv 0$ in $T_{\varepsilon}$. Then we compute

$$
\begin{aligned}
\int_{\Omega} \sigma_{i j}\left(z_{\varepsilon}^{m}\right) e_{i j}\left(z_{\varepsilon}^{l}\right) d x= & \sum_{k} \int_{C_{\varepsilon}^{k}} \sigma_{i j}\left(w_{\varepsilon}^{m k}\right) e_{i j}\left(w_{\varepsilon}^{l k}\right)\left(\varphi_{\varepsilon}^{k}\right)^{2} d x \\
& -2 \sum_{k} \int_{C_{\varepsilon}^{k} \cap\left\{s_{\varepsilon} / 2<R_{\varepsilon}^{k}<s_{\varepsilon}\right\}} \sigma_{i j}\left(w_{\varepsilon}^{m k}\right) \frac{\partial \varphi_{\varepsilon}^{k}}{\partial x_{i}}\left(e_{l}-w_{\varepsilon}^{l k}\right)_{j} d x \\
& +\sum_{k} \int_{C_{\varepsilon}^{k} \cap\left\{s_{\varepsilon} / 2<R_{\varepsilon}^{k}<s_{\varepsilon}\right\}}\left(e_{m}-w_{\varepsilon}^{m k}\right)_{i} \frac{\partial \varphi_{\varepsilon}^{k}}{\partial x_{i}}\left(e_{l}-w_{\varepsilon}^{l k}\right)_{j} \frac{\partial \varphi_{\varepsilon}^{k}}{\partial x_{j}} d x .
\end{aligned}
$$

Thanks to Lemma 4 and to the definition of $\varphi_{\varepsilon}^{k}$, one can prove that the two last sums are respectively bounded by : $C\left|\ln s_{\varepsilon}\right| /\left(\varepsilon^{2} \ln ^{2} r_{\varepsilon}\right)$ and $C \ln ^{2} s_{\varepsilon} /\left(\varepsilon^{2} \ln ^{2} r_{\varepsilon}\right)$. These two upper bounds converge to 0 , because $\gamma$ is finite and thanks to the choice of $s_{\varepsilon}$. Moreover, the first term of the preceding equality can be computed as

$$
\begin{gathered}
\int_{C_{\varepsilon}^{k}} \sigma_{i j}\left(w_{\varepsilon}^{m k}\right) e_{i j}\left(w_{\varepsilon}^{l k}\right)\left(\varphi_{\varepsilon}^{k}\right)^{2} d x=\int_{C_{\varepsilon}^{k}} \sigma_{i j}\left(w_{\varepsilon}^{m k}\right) e_{i j}\left(w_{\varepsilon}^{l k}\right) d x \\
+\int_{C_{\varepsilon}^{k} \cap\left\{s_{\varepsilon} / 2<R_{\varepsilon}^{k}<s_{\varepsilon}\right\}} \sigma_{i j}\left(w_{\varepsilon}^{m k}\right) e_{i j}\left(w_{\varepsilon}^{l k}\right)\left(\left(\varphi_{\varepsilon}^{k}\right)^{2}-1\right) d x
\end{gathered}
$$

and using the definition (12) of $\varphi_{\varepsilon}^{k}$ we get

$$
\begin{aligned}
\mid \int_{C_{\varepsilon}^{k} \cap\left\{s_{\varepsilon} / 2<R_{\varepsilon}^{k}<s_{\varepsilon}\right\}} & \sigma_{i j}\left(w_{\varepsilon}^{m k}\right) e_{i j}\left(w_{\varepsilon}^{l k}\right)\left(\left(\varphi_{\varepsilon}^{k}\right)^{2}-1\right) d x \mid \\
\leq & \int_{C_{\varepsilon}^{k} \cap\left\{s_{\varepsilon} / 2<R_{\varepsilon}^{k}<s_{\varepsilon}\right\}} \sigma_{i j}\left(w_{\varepsilon}^{m k}\right) e_{i j}\left(w_{\varepsilon}^{l k}\right) d x .
\end{aligned}
$$

Thanks to the estimates of Lemma 4, we deduce

$$
\lim _{\varepsilon \rightarrow 0} \sum_{k} \int_{C_{\varepsilon}^{k} \cap\left\{s_{\varepsilon} / 2<R_{\varepsilon}^{k}<s_{\varepsilon}\right\}} \sigma_{i j}\left(w_{\varepsilon}^{m k}\right) e_{i j}\left(w_{\varepsilon}^{l k}\right) d x=0,
$$

which implies

$$
\lim _{\varepsilon \rightarrow 0} \int_{\Omega} \sigma_{i j}\left(z_{\varepsilon}^{m}\right) e_{i j}\left(z_{\varepsilon}^{l}\right) d x=\lim _{\varepsilon \rightarrow 0} \int_{C_{\varepsilon}} \sigma_{i j}\left(w_{\varepsilon}^{m k}\right) e_{i j}\left(w_{\varepsilon}^{l k}\right) d x .
$$

One concludes using the first assertion. Because $\left(z_{\varepsilon}^{m}\right)_{\mid \Gamma_{1}}=0$, there exists some positive constant $C$ such that

$$
\int_{\Omega}\left|\nabla z_{\varepsilon}^{m}\right|^{2} d x \leq C \int_{\Omega} \sigma_{i j}\left(z_{\varepsilon}^{m}\right) e_{i j}\left(z_{\varepsilon}^{m}\right) d x
$$

Hence $\left(z_{\varepsilon}^{m}\right)_{\varepsilon}$ is bounded in $H^{1}\left(\Omega, \mathbf{R}^{3}\right)$, which implies that a subsequence still denoted $\left(z_{\varepsilon}^{m}\right)_{\varepsilon}$ converges to some $z^{*}$ in the weak topology of $H^{1}\left(\Omega, \mathbf{R}^{3}\right)$ and in the strong topology of $L^{2}\left(\Omega, \mathbf{R}^{3}\right)$. We observe that $z_{\varepsilon}^{m}=0$ in $\Omega \backslash \overline{B_{\varepsilon}}$ and because the sequence of characteristic functions of $\Omega \backslash \overline{B_{\varepsilon}}$ converges to 1 in the strong topology of $L^{2}(\Omega)$, we infer that $z^{*}=0$. Hence $\left(z_{\varepsilon}^{m}\right)_{\varepsilon}$ converges to 0 in the weak topology of $H^{1}\left(\Omega, \mathbf{R}^{3}\right)$. 


\section{Convergence}

We define the topology $\tau$ which will be used throughout this paragraph as

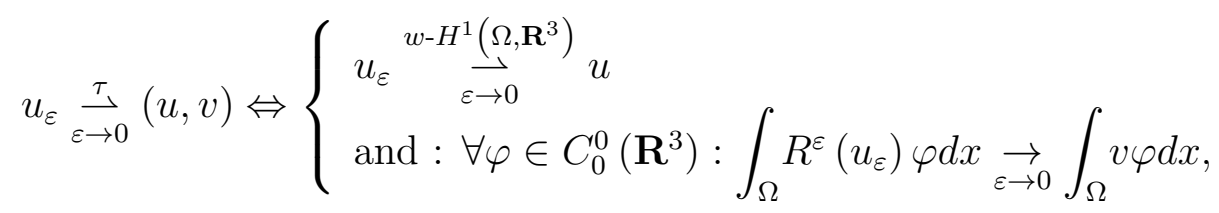

where $w-H^{1}\left(\Omega, \mathbf{R}^{3}\right)$ stands for the weak topology of $H^{1}\left(\Omega, \mathbf{R}^{3}\right)$ and $R^{\varepsilon}$ is the rescaled restriction operator defined in (8) .

Our main result reads as follows

Theorem 6 Suppose that $\gamma=\lim _{\varepsilon \rightarrow 0}\left(-1 /\left(\varepsilon^{2} \ln r_{\varepsilon}\right)\right)$ is finite, $\lambda_{o}$ and $\mu_{o}$ are finite and $\mu_{o}$ is positive. Then, the sequence $\left(F^{\varepsilon}\right)_{\varepsilon}$ epi-converges in the topology $\tau$ to the functional $F^{o}$ defined on $H^{1}\left(\Omega, \mathbf{R}^{3}\right) \times L^{1}\left(\Omega, \mathbf{R}^{3}\right)$ by:

$$
F^{o}(u, v)=\left\{\begin{array}{r}
\int_{\Omega} \sigma_{i j}(u) e_{i j}(u) d x+2 \pi \gamma \int_{\Omega}(v-u)^{t} A(v-u) d x+\pi E_{o} \int_{\Omega}\left(e_{33}(v)\right)^{2} d x \\
+\infty \\
\text { if }(u, v) \in H_{\Gamma_{1}}^{1}\left(\Omega, \mathbf{R}^{3}\right) \times V \\
\text { otherwise }
\end{array}\right.
$$

using the summation convention with respect to repeated indices and where $A$ is the diagonal matrix with : $A_{11}=\mu(1+\kappa) / \kappa=A_{22}$ and $A_{33}=\mu$, where $\kappa=(\lambda+3 \mu) /(\lambda+\mu)$, $E_{o}=\mu_{o}\left(3 \lambda_{o}+2 \mu_{o}\right) /\left(\lambda_{o}+\mu_{o}\right)$ and $V$ denotes the subspace

$$
V=\left\{v \in L^{2}\left(\Omega, \mathbf{R}^{3}\right) \mid v_{3 \mid \Gamma_{1}}=0, e_{33}(v) \in L^{2}(\Omega)\right\} .
$$

As a consequence of this theorem and of the properties of the epi-convergence (see [1] for a definition and the main properties of this notion of convergence well-fitted to the description of the asymptotic behaviour of the solution of minimization problems), one gets the following asymptotic behaviour, when $\varepsilon$ goes to 0 , of the solution $u^{\varepsilon}$ of (5)

Corollary 7 Under the hypotheses of Theorem [6, the solution $u^{\varepsilon}$ of (5) converges, in the topology $\tau$, to the solution $\left(u^{o}, v^{o}\right)$ in the space $H_{\Gamma_{1}}^{1}\left(\Omega, \mathbf{R}^{3}\right) \times V$ of the following problem

$$
\left\{\begin{aligned}
-\sigma_{i j, j}\left(u^{o}\right)-2 \gamma \pi A_{i j}\left(v^{o}-u^{o}\right)_{j} & =f_{i} & & \text { in } \Omega, i=1,2,3 \\
u^{o} & =0 & & \text { on } \Gamma_{1} \\
\sigma_{i j}\left(u^{o}\right) n_{j} & =0 & & \text { on } \partial \omega \times] 0, L\left[\cup \Gamma_{2}\right. \\
E_{o} \frac{\partial}{\partial x_{3}}\left(e_{33}\left(v^{o}\right)\right) & =2 \gamma \mu\left(v^{o}-u^{o}\right)_{3} & & \text { in } \Omega \\
v^{o} & =0 & & i, j=1,2,3 \\
\left(u^{o}\right)_{\alpha} & =\left(v^{o}\right)_{\alpha} & & \text { on } \Gamma_{1} \\
e_{33}\left(v^{o}\right) & =0 & & \text { in } \Omega, \alpha=1,2 \\
& & & \text { on } \Gamma_{2} .
\end{aligned}\right.
$$


$\left(u^{o}, v^{o}\right)$ is the unique solution of the minimization problem

$$
\min \left\{F^{o}(u, v)-2 \int_{\Omega} f . u d x \mid u \in H_{\Gamma_{1}}^{1}\left(\Omega, \mathbf{R}^{3}\right), v \in V\right\}
$$

Moreover, the convergence of the linked energies : $\lim _{\varepsilon \rightarrow 0} F^{\varepsilon}\left(u^{\varepsilon}\right)=F^{o}\left(u^{o}, v^{o}\right)$ holds true.

Remark 8 In the expression of the limit functional $F^{o}$, the term $\pi E_{o} \int_{\Omega}\left(e_{33}(v)\right)^{2} d x$ can be interpreted as the "pure influence" of the fibers, due to their longitudinal repartition, on the asymptotic behaviour. The term $2 \pi \gamma \int_{\Omega}(v-u)^{t} A(v-u) d x$ can be interpreted as the mixed influence of the fibers and of the elastic material (for example, shearing effect of the fibers on the material, for the term $\left.2 \pi \gamma \mu \int_{\Omega}\left(v_{3}-u_{3}\right)^{2} d x\right)$.

of Theorem 6. This proof will be decomposed in two main parts, corresponding to the verification of the two assertions of the epi-convergence. As a first step, let us verify : For every $u$ in $H_{\Gamma_{1}}^{1}\left(\Omega, \mathbf{R}^{3}\right)$ and for every $v$ in $V$, there exists a sequence $\left(u_{\varepsilon}^{o}\right)_{\varepsilon}$ of elements of $H_{\Gamma_{1}}^{1}\left(\Omega, \mathbf{R}^{3}\right)$ converging to $(u, v)$ in the topology $\tau$ and such that : $\lim _{\sup _{\varepsilon \rightarrow 0}} F^{\varepsilon}\left(u_{\varepsilon}^{o}\right) \leq$ $F^{o}(u, v)$.

Let us first choose any element $u$ of $C^{1}\left(\bar{\Omega}, \mathbf{R}^{3}\right) \cap H_{\Gamma_{1}}^{1}\left(\Omega, \mathbf{R}^{3}\right)$ and any element $v$ of $C^{2}\left(\bar{\Omega}, \mathbf{R}^{3}\right) \cap V$. For every $k=\left(k_{1}, k_{2}\right)$, we define the function $\mathcal{R}_{\varepsilon}(v)$ in $B_{\varepsilon}^{k}$ by its three components as follows:

$$
\left\{\begin{aligned}
\left(\mathcal{R}_{\varepsilon}(v)\right)_{\alpha}\left(x_{1}, x_{2}, x_{3}\right)= & v_{\alpha}\left(k_{1} \varepsilon, k_{2} \varepsilon, x_{3}\right) \\
& -\frac{\lambda^{\varepsilon}}{2\left(\mu^{\varepsilon}+\lambda^{\varepsilon}\right)}\left(x_{\alpha}-k_{\alpha} \varepsilon\right) \frac{\partial v_{3}}{\partial x_{3}}\left(k_{1} \varepsilon, k_{2} \varepsilon, x_{3}\right) \\
\left(\mathcal{R}_{\varepsilon}(v)\right)_{3}\left(x_{1}, x_{2}, x_{3}\right)= & v_{3}\left(k_{1} \varepsilon, k_{2} \varepsilon, x_{3}\right)-\left(x_{1}-k_{1} \varepsilon\right) \frac{\partial v_{1}}{\partial x_{3}}\left(k_{1} \varepsilon, k_{2} \varepsilon, x_{3}\right) \\
& -\left(x_{2}-k_{2} \varepsilon\right) \frac{\partial v_{2}}{\partial x_{3}}\left(k_{1} \varepsilon, k_{2} \varepsilon, x_{3}\right) .
\end{aligned}\right.
$$

Let us choose some smooth function $\psi_{\varepsilon}$ identically equal to 1 (resp. to 0 ) in $\Omega \backslash \overline{\Sigma_{2 \varepsilon}}$ (resp. in $\Sigma_{\varepsilon}$ ), with : $\Sigma_{\varepsilon}=\left\{x \in \Omega \mid d\left(x, \Gamma_{1}\right)<\varepsilon\right\}$. We define:

$$
\begin{aligned}
u_{\varepsilon}^{o} & =\left(1-\psi_{\varepsilon}\right) u+\psi_{\varepsilon}\left(\left(e_{m}-z_{\varepsilon}^{m}\right) u_{m}+z_{\varepsilon}^{m}\left(\mathcal{R}_{\varepsilon}(v)\right)_{m}\right) \\
& =u-\psi_{\varepsilon} z_{\varepsilon}^{m}\left(u_{m}-\left(\mathcal{R}_{\varepsilon}(v)\right)_{m}\right),
\end{aligned}
$$

where $u_{m}$ and $\left(\mathcal{R}_{\varepsilon}(v)\right)_{m}$ are the $m$-th components of $u$ and $\mathcal{R}_{\varepsilon}(v)$ in the canonical basis $\left(e_{m}\right)_{m=1,2,3}$ of $\mathbf{R}^{3}$ and $z_{\varepsilon}^{m}$ is defined in (13). One has the following estimates.

Lemma 9 1. There exists some positive constant $C$ independant of $\varepsilon$ such that

$$
\begin{aligned}
\left|u_{\varepsilon}^{o}\right|(x) & \leq C \quad \forall x \in \Omega \\
\left|\nabla \mathcal{R}_{\varepsilon}(v)\right|(x) & \leq C \quad \forall x \in B_{\varepsilon} \\
\left|\mathcal{R}_{\varepsilon}(v)-v\right|(x) & \leq C r_{\varepsilon} \quad \forall x \in T_{\varepsilon} \\
\left|\mathcal{R}_{\varepsilon}(v)-v\right|(x) & \leq C s_{\varepsilon} \quad \forall x \in B_{\varepsilon} .
\end{aligned}
$$


2. $u_{\varepsilon}^{o}$ belongs to $H_{\Gamma_{1}}^{1}\left(\Omega, \mathbf{R}^{3}\right)$, $\left(u_{\varepsilon}^{o}\right)_{\varepsilon}$ converges to $(u, v)$ in the above defined topology $\tau$.

Proof. 1. Because $v$ belongs to $L^{\infty}\left(\Omega, \mathbf{R}^{3}\right)$, together with its first order derivatives, we get, in every $B_{\varepsilon}^{k}:\left|\mathcal{R}_{\varepsilon}(v)\right| \leq C$ and $\left|\nabla \mathcal{R}_{\varepsilon}(v)\right| \leq C^{\prime}$, where $C$ and $C^{\prime}$ are positive constants. Using Lemma 4 , we get : $\left|u_{\varepsilon}^{o}\right| \leq C$, in $\Omega$. One has, for every $k=\left(k_{1}, k_{2}\right)$

$$
\begin{aligned}
\left|\left(\mathcal{R}_{\varepsilon}(v)-v\right)_{\alpha}\right|_{\mid T_{\varepsilon}^{k}} \leq & \left|v_{\alpha}\left(k_{1} \varepsilon, k_{2} \varepsilon, x_{3}\right)-v_{\alpha}\left(x_{1}, x_{2}, x_{3}\right)\right| \\
& \quad+\frac{\lambda^{\varepsilon}}{2\left(\mu^{\varepsilon}+\lambda^{\varepsilon}\right)}\left|\left(x_{\alpha}-k_{\alpha} \varepsilon\right) \frac{\partial v_{3}}{\partial x_{3}}\left(k_{1} \varepsilon, k_{2} \varepsilon, x_{3}\right)\right| \\
\leq & C r_{\varepsilon},
\end{aligned}
$$

because $v$ belongs to $C^{1}\left(\bar{\Omega}, \mathbf{R}^{3}\right)$ and using the hypotheses on $\lambda^{\varepsilon}$ and $\mu^{\varepsilon}$. Similarly, we have : $\left|\left(\mathcal{R}_{\varepsilon}(v)-v\right)_{3}\right|_{\mid T_{\varepsilon}^{k}} \leq C r_{\varepsilon}$, and : $\left|\mathcal{R}_{\varepsilon}(v)-v\right|_{\mid B_{\varepsilon}^{k}} \leq C s_{\varepsilon}$, for every $k$.

2. Observe that $u_{\varepsilon}^{o}$ belongs to $H_{\Gamma_{1}}^{1}\left(\Omega, \mathbf{R}^{3}\right)$ because $u$ vanishes on $\Gamma_{1}$ and $\psi_{\varepsilon}$ also vanishes on $\Gamma_{1}$. Furthermore, there exists some constant $C_{m}$ such that one has in $B_{\varepsilon}$

$$
\begin{aligned}
\left|\nabla u_{\varepsilon}^{o}\right| & \leq\left|\nabla u_{m}\left(e_{m}-z_{\varepsilon}^{m}\right)+z_{\varepsilon}^{m} \nabla\left(\mathcal{R}_{\varepsilon}(v)\right)_{m}+\left(\left(\mathcal{R}_{\varepsilon}(v)\right)_{m}-u_{m}\right) \nabla z_{\varepsilon}^{m}\right| \\
& \leq C_{m}\left(\left|\nabla u_{m}\right|+\varepsilon\left|\nabla z_{\varepsilon}^{m}\right|+\left|\nabla z_{\varepsilon}^{m}\right|\left|v_{m}-u_{m}\right|\right),
\end{aligned}
$$

for some constant $C_{m}$, thanks to the preceding estimates. We then compute

$$
\int_{\Omega}\left|\nabla u_{\varepsilon}^{o}\right|^{2} d x=\int_{\Omega \backslash \overline{B_{\varepsilon}}}\left|\nabla u_{\varepsilon}^{o}\right|^{2} d x+\int_{B_{\varepsilon}}\left|\nabla u_{\varepsilon}^{o}\right|^{2} d x
$$

Thanks to (17) and to Lemma 5 one has

$$
\begin{aligned}
\int_{B_{\varepsilon}}\left|\nabla u_{\varepsilon}^{o}\right|^{2} d x & \leq C_{m}^{\prime}\left(\int_{B_{\varepsilon}}\left|\nabla u_{m}\right|^{2} d x+\varepsilon \int_{B_{\varepsilon}}\left|\nabla z_{\varepsilon}^{m}\right|^{2} d x+\int_{B_{\varepsilon}}\left|v_{m}-u_{m}\right|^{2}\left|\nabla z_{\varepsilon}^{m}\right|^{2} d x\right) \\
& \leq C,
\end{aligned}
$$

where $C$ is some positive constant independant of $\varepsilon$. Furthermore, because $z_{\varepsilon}^{m}$ outside $B_{\varepsilon}$

$$
\int_{\Omega \backslash \overline{B_{\varepsilon}}}\left|\nabla u_{\varepsilon}^{o}\right|^{2} d x \underset{\varepsilon \rightarrow 0}{\rightarrow} \int_{\Omega}|\nabla u|^{2} d x .
$$

This proves that $\left(u_{\varepsilon}^{o}\right)_{\varepsilon}$ converges to $u$ in the weak topology of $H^{1}\left(\Omega, \mathbf{R}^{3}\right)$. Let $\varphi$ be any element of $C_{0}^{1}\left(\mathbf{R}^{3}, \mathbf{R}^{3}\right)$. We have, because : $\left(z_{\varepsilon}^{m}\right)_{\mid T_{\varepsilon}}=e_{m}$

$$
\begin{aligned}
\int_{\Omega} \varphi R^{\varepsilon}\left(u_{\varepsilon}^{o}\right) d x & =\frac{|\Omega|}{\left|T_{\varepsilon}\right|} \int_{T_{\varepsilon}} \varphi u_{\varepsilon}^{o} d x \\
& =\frac{|\Omega|}{\left|T_{\varepsilon}\right|} \int_{T_{\varepsilon}} \varphi \mathcal{R}_{\varepsilon}(v) d x \\
& =\frac{|\Omega|\left|T_{\varepsilon}^{k} \cap \omega\right|}{\left|T_{\varepsilon}\right| \varepsilon^{2}} \sum_{k} \varepsilon^{2} \int_{0}^{L} \varphi\left(k_{1} \varepsilon, k_{2} \varepsilon, x_{3}\right) v\left(k_{1} \varepsilon, k_{2} \varepsilon, x_{3}\right) d x_{3}+o_{\varepsilon}
\end{aligned}
$$


$\varphi$ and $v$ being continuously differentiable and $\left|T_{\varepsilon}^{k} \cap \omega\right|$ being independant of $k$. We have, thanks to the smoothness of $\varphi$ and $v$

$$
\lim _{\varepsilon \rightarrow 0} \sum_{k} \varepsilon^{2} \int_{0}^{L} \varphi\left(k_{1} \varepsilon, k_{2} \varepsilon, x_{3}\right) v\left(k_{1} \varepsilon, k_{2} \varepsilon, x_{3}\right) d x_{3}=\int_{\Omega} \varphi v d x
$$

and we observe that : $\lim _{\varepsilon \rightarrow 0}\left(|\Omega|\left|T_{\varepsilon}^{k} \cap \omega\right|\right) /\left(\left|T_{\varepsilon}\right| \varepsilon^{2}\right)=1$. This proves that the sequence $\left(u_{\varepsilon}^{o}\right)_{\varepsilon}$ converges to $(u, v)$ in the above defined topology $\tau$.

For every $u$ in $C^{1}\left(\bar{\Omega}, \mathbf{R}^{3}\right) \cap H_{\Gamma_{1}}^{1}\left(\Omega, \mathbf{R}^{3}\right)$ and every $v$ in $C^{1}\left(\bar{\Omega}, \mathbf{R}^{3}\right)$, we compute

$$
\begin{aligned}
F^{\varepsilon}\left(u_{\varepsilon}^{o}\right)=\int_{\Omega \backslash \overline{C_{\varepsilon} \cup T_{\varepsilon}}} \sigma_{i j}(u) e_{i j}(u) & d x+\int_{C_{\varepsilon}} \sigma_{i j}\left(u_{\varepsilon}^{o}\right) e_{i j}\left(u_{\varepsilon}^{o}\right) d x \\
& +\int_{T_{\varepsilon}} \sigma_{i j}^{\varepsilon}\left(\mathcal{R}_{\varepsilon}(v)\right) e_{i j}\left(\mathcal{R}_{\varepsilon}(v)\right) d x .
\end{aligned}
$$

Because the characteristic function of $\Omega \backslash \overline{C_{\varepsilon} \cup T_{\varepsilon}}$ converges to 1 in the strong topology of $L^{2}(\Omega)$, the first integral of (19) immediately leads to

$$
\lim _{\varepsilon \rightarrow 0} \int_{\Omega \backslash \overline{C_{\varepsilon} \cup T_{\varepsilon}}} \sigma_{i j}(u) e_{i j}(u) d x=\int_{\Omega} \sigma_{i j}(u) e_{i j}(u) d x .
$$

Let us study the second integral of (19). One has, using the definition (16) of the test-function $u_{\varepsilon}^{o}$

$$
\begin{aligned}
\int_{C_{\varepsilon}} \sigma_{i j}\left(u_{\varepsilon}^{o}\right) e_{i j}\left(u_{\varepsilon}^{o}\right) d x & \\
=\int_{C_{\varepsilon}} \sigma_{i j}(u) & e_{i j}(u) d x+2 \int_{C_{\varepsilon}} \sigma_{i j}(u) e_{i j}\left(z_{\varepsilon}^{m}\left(\left(\mathcal{R}_{\varepsilon}(v)\right)_{m}-u_{m}\right)\right) d x \\
& \quad+\int_{C_{\varepsilon}} \sigma_{i j}\left(z_{\varepsilon}^{m}\left(\left(\mathcal{R}_{\varepsilon}(v)\right)_{m}-u_{m}\right)\right) e_{i j}\left(z_{\varepsilon}^{l}\left(\left(\mathcal{R}_{\varepsilon}(v)\right)_{l}-u_{l}\right)\right) d x
\end{aligned}
$$

The second integral of the right hand side of (21) converges to 0 , because $\left(z_{\varepsilon}^{m}\right)_{\varepsilon}$ converges to 0 in the weak topology of $H_{\Gamma_{1}}^{1}\left(\Omega, \mathbf{R}^{3}\right)$ and thanks to the estimates of Lemma 8 , The third integral of this right hand side of (21) can be computed as

$$
\begin{aligned}
\int_{C_{\varepsilon}} \sigma_{i j}\left(z_{\varepsilon}^{m}\left(v_{m}-u_{m}\right)\right) e_{i j}\left(z_{\varepsilon}^{l}\left(v_{l}-u_{l}\right)\right) d x \\
\quad+2 \int_{C_{\varepsilon}} \sigma_{i j}\left(z_{\varepsilon}^{m}\left(\left(\mathcal{R}_{\varepsilon}(v)\right)_{m}-u_{m}\right)\right) e_{i j}\left(z_{\varepsilon}^{l}\left(v_{l}-u_{l}\right)\right) d x \\
\quad+\int_{C_{\varepsilon}} \sigma_{i j}\left(z_{\varepsilon}^{m}\left(\left(\mathcal{R}_{\varepsilon}(v)\right)_{m}-v_{m}\right)\right) e_{i j}\left(z_{\varepsilon}^{l}\left(\left(\mathcal{R}_{\varepsilon}(v)\right)_{l}-v_{l}\right)\right) d x
\end{aligned}
$$

Thanks to Lemmas 5 and 9, the two last integrals of (22) converge to 0 and the first integral of (22) is equal to

$$
\int_{\Omega} \sigma_{i j}\left(z_{\varepsilon}^{m}\right) e_{i j}\left(z_{\varepsilon}^{l}\right)\left(v_{m}-u_{m}\right)\left(v_{l}-u_{l}\right) d x+o_{\varepsilon},
$$


with $\lim _{\varepsilon \rightarrow 0} O_{\varepsilon}=0$, because $\left(z_{\varepsilon}^{m}\right)_{\varepsilon}$ converges to 0 in the weak topology of $H_{\Gamma_{1}}^{1}\left(\Omega, \mathbf{R}^{3}\right)$. One deduces from Lemma 5 and the smoothness of $u$ and $v$ that

$$
\lim _{\varepsilon \rightarrow 0} \int_{\Omega} \sigma_{i j}\left(z_{\varepsilon}^{m}\right) e_{i j}\left(z_{\varepsilon}^{l}\right)\left(v_{m}-u_{m}\right)\left(v_{l}-u_{l}\right) d x=2 \pi \gamma \int_{\Omega}(v-u)^{t} A(v-u) d x .
$$

In order to study the third integral of (19), one observes that the above expression of $\mathcal{R}_{\varepsilon}(v)$ implies

$$
\begin{aligned}
\operatorname{Tr}\left(e\left(\mathcal{R}_{\varepsilon}(v)\right)\right) & =\frac{\mu^{\varepsilon}}{\mu^{\varepsilon}+\lambda^{\varepsilon}} \frac{\partial v_{3}}{\partial x_{3}}\left(k_{1} \varepsilon, k_{2} \varepsilon, x_{3}\right)-\left(x_{\alpha}-k_{\alpha} \varepsilon\right) \frac{\partial^{2} v_{\alpha}}{\partial x_{3}^{2}}\left(k_{1} \varepsilon, k_{2} \varepsilon, x_{3}\right) \\
\sigma_{11}^{\varepsilon}\left(\mathcal{R}_{\varepsilon}(v)\right) & =-\lambda^{\varepsilon}\left(x_{\alpha}-k_{\alpha} \varepsilon\right) \frac{\partial^{2} v_{\alpha}}{\partial x_{3}^{2}}\left(k_{1} \varepsilon, k_{2} \varepsilon, x_{3}\right) \\
\sigma_{22}^{\varepsilon}\left(\mathcal{R}_{\varepsilon}(v)\right) & =-\lambda^{\varepsilon}\left(x_{\alpha}-k_{\alpha} \varepsilon\right) \frac{\partial^{2} v_{\alpha}}{\partial x_{3}^{2}}\left(k_{1} \varepsilon, k_{2} \varepsilon, x_{3}\right) \\
\sigma_{12}^{\varepsilon}\left(\mathcal{R}_{\varepsilon}(v)\right) & =0 \\
\sigma_{33}^{\varepsilon}\left(\mathcal{R}_{\varepsilon}(v)\right) & =\mu^{\varepsilon} \frac{2 \mu^{\varepsilon}+3 \lambda^{\varepsilon}}{\mu^{\varepsilon}+\lambda^{\varepsilon}} \frac{\partial v_{3}}{\partial x_{3}}\left(k_{1} \varepsilon, k_{2} \varepsilon, x_{3}\right) \\
\sigma_{\alpha 3}^{\varepsilon}\left(\mathcal{R}_{\varepsilon}(v)\right) & =-\mu^{\varepsilon}\left(\mu^{\varepsilon}+\lambda^{\varepsilon}\right)\left(x_{\alpha}-k_{\alpha} \varepsilon\right) \frac{\partial^{2} v_{\alpha}}{\partial x_{3}^{2}}\left(k_{1} \varepsilon, k_{2} \varepsilon, x_{3}\right) .
\end{aligned}
$$

One easily proves that all the terms of the third integral of (19) converge to 0 except the following one

$$
\begin{aligned}
\int_{T_{\varepsilon}} \sigma_{33}^{\varepsilon} & \left(\mathcal{R}_{\varepsilon}(v)\right) e_{33}\left(\mathcal{R}_{\varepsilon}(v)\right) d x \\
& =\frac{\pi \mu^{\varepsilon}\left(r_{\varepsilon}\right)^{2}}{\varepsilon^{2}} \frac{2 \mu^{\varepsilon}+3 \lambda^{\varepsilon}}{\mu^{\varepsilon}+\lambda^{\varepsilon}} \sum_{k} \varepsilon^{2} \int_{0}^{L}\left(\frac{\partial v_{3}}{\partial x_{3}}\right)^{2}\left(k_{1} \varepsilon, k_{2} \varepsilon, x_{3}\right) d x_{3}+o_{\varepsilon} \\
& \rightarrow \pi E_{o} \int_{\Omega}\left(e_{33}(v)\right)^{2} d x,
\end{aligned}
$$

with the above definition of $E_{o}$. Thus, we get, for this third integral of (19)

$$
\lim _{\varepsilon \rightarrow 0} \int_{T_{\varepsilon}} \sigma_{i j}^{\varepsilon}\left(\mathcal{R}_{\varepsilon}(v)\right) e_{i j}\left(\mathcal{R}_{\varepsilon}(v)\right) d x=\pi E_{o} \int_{\Omega}\left(e_{33}(v)\right)^{2} d x
$$

From (20), (23) and (24), we thus derive : $\lim _{\varepsilon \rightarrow 0} F^{\varepsilon}\left(u_{\varepsilon}^{o}\right)=F^{o}(u, v)$.

We conclude the verification of this first assertion, using a density argument and the diagonalization argument contained in [1, Corollary 1.18]. Indeed, for every $u$ in $H_{\Gamma_{1}}^{1}\left(\Omega, \mathbf{R}^{3}\right)$, there exists a sequence $\left(u^{n}, v^{n}\right)_{n}$ in $\left(C^{1}\left(\bar{\Omega}, \mathbf{R}^{3}\right) \cap H_{\Gamma_{1}}^{1}\left(\Omega, \mathbf{R}^{3}\right)\right) \times\left(C^{2}\left(\bar{\Omega}, \mathbf{R}^{3}\right) \cap V\right)$ converging to $(u, v)$ in the strong topology of the space $H^{1}\left(\Omega, \mathbf{R}^{3}\right) \times V$. Thanks to Lemma 9. $\left(\left(u^{n}\right)_{\varepsilon}^{o}\right)_{\varepsilon}$ converges to $\left(u^{n}, v^{n}\right)$ in the topology $\tau$ and

$$
\lim _{n \rightarrow+\infty} \lim _{\varepsilon \rightarrow 0} F^{\varepsilon}\left(\left(u^{n}\right)_{\varepsilon}^{o}\right)=\lim _{n \rightarrow+\infty} F^{o}\left(u^{n}, v^{n}\right)=F^{o}(u, v) .
$$


The space $H^{1}\left(\Omega, \mathbf{R}^{3}\right) \times L^{1}\left(\Omega, \mathbf{R}^{3}\right)$ is metrizable for the topology $\tau$. One deduces from [1, Corollary 1.18], the existence of a subsequence $\left(\left(u^{n(\varepsilon)}\right)_{\varepsilon}^{o}\right)_{\varepsilon}$ converging to $u$ in the weak topology of $H_{\Gamma_{1}}^{1}\left(\Omega, \mathbf{R}^{3}\right)$, such that $\left(R^{\varepsilon}\left(v^{n(\varepsilon)}\right)\right)_{\varepsilon}$ converges to $v$ in the weak* topology of $L^{1}\left(\Omega, \mathbf{R}^{3}\right)$ and $: \limsup _{\varepsilon \rightarrow 0} F^{\varepsilon}\left(\left(u^{n(\varepsilon)}\right)_{\varepsilon}^{o}\right) \leq F^{o}(u, v)$. This ends the verification of the first assertion.

Let us now prove the second assertion of the epi-convergence, that is : For every sequence $\left(u_{\varepsilon}\right)_{\varepsilon}$ of elements of $H_{\Gamma_{1}}^{1}\left(\Omega, \mathbf{R}^{3}\right)$, converging to $(u, v)$ in the topology $\tau$, then $v$ belongs to $V$, satisfies : $v=0$, on $\Gamma_{1}$, and $: \liminf _{\varepsilon \rightarrow 0} F^{\varepsilon}\left(u_{\varepsilon}\right) \geq F^{o}(u, v)$.

Let $\left(u^{n}\right)_{n}$ be any sequence of smooth functions in $C^{1}\left(\bar{\Omega}, \mathbf{R}^{3}\right) \cap H_{\Gamma_{1}}^{1}\left(\Omega, \mathbf{R}^{3}\right)$ converging to $u$ in the strong topology of $H^{1}\left(\Omega, \mathbf{R}^{3}\right)$ and $\left(v^{n}\right)_{n}$ be any sequence of smooth functions in $C^{2}\left(\bar{\Omega}, \mathbf{R}^{3}\right) \cap V$ converging to $v$ in the strong topology of $V$. Let us suppose that $\sup _{\varepsilon} F^{\varepsilon}\left(u_{\varepsilon}\right)<+\infty$, otherwise the assertion is trivially satisfied. Under these hypotheses, one proves

Lemma $10\left(u_{\varepsilon}\right)_{\varepsilon}$ is bounded in $H_{\Gamma_{1}}^{1}\left(\Omega, \mathbf{R}^{3}\right)$ and the sequence $\left(R^{\varepsilon}\left(u_{\varepsilon}\right)\right)_{\varepsilon}$ converges in the weak $k^{*}$ topology of $L^{1}\left(\Omega, \mathbf{R}^{3}\right)$ to some $v$ belonging to $V$.

Proof. We use some argument similar to [2, Lemme A1], defining:

$$
\Phi_{\varepsilon}=e_{33}\left(u_{\varepsilon}\right), \delta_{\varepsilon}=\frac{|\Omega|}{\left|T_{\varepsilon}\right|} \mathbf{1}_{T_{\varepsilon}} d x, \delta=\mathbf{1}_{\Omega} d x
$$

$\delta_{\varepsilon}$ and $\delta$ are two bounded Radon measures such that $\left(\delta_{\varepsilon}\right)_{\varepsilon}$ converges weakly to $\delta$ in the sense of measures. We then compute

$$
\begin{aligned}
\int_{\mathbf{R}^{3}}\left|\Phi_{\varepsilon}\right| \delta_{\varepsilon} & \leq\left(\int_{\mathbf{R}^{3}}\left|\Phi_{\varepsilon}\right|^{2} \delta_{\varepsilon}\right)^{1 / 2} \sqrt{\left|T_{\varepsilon}\right|} \\
& \leq \frac{C}{\sqrt{\left|T_{\varepsilon}\right|}}\left(\int_{T_{\varepsilon}}\left|\Phi_{\varepsilon}\right|^{2} d x\right)^{1 / 2} \leq C\left(\sup _{\varepsilon} F^{\varepsilon}\left(u_{\varepsilon}\right)\right)^{1 / 2}<+\infty,
\end{aligned}
$$

because $\left(\lambda^{\varepsilon}\left|T_{\varepsilon}\right|\right)_{\varepsilon}$ and $\left(\mu^{\varepsilon}\left|T_{\varepsilon}\right|\right)_{\varepsilon}$ have finite limits. Hence, the sequence $\left(\Phi_{\varepsilon} \delta_{\varepsilon}\right)_{\varepsilon}$ of measures has uniformly bounded variations. One can extract some subsequence, still denoted by $\left(\Phi_{\varepsilon} \delta_{\varepsilon}\right)_{\varepsilon}$, which converges to some measure $\Phi$. For every $\varphi$ in $C_{c}^{o}\left(\mathbf{R}^{3}\right)$, we write Fenchel's inequality

$$
\int_{\mathbf{R}^{3}}\left|\Phi_{\varepsilon}\right|^{2} \delta_{\varepsilon} \geq 2 \int_{\mathbf{R}^{3}} \Phi_{\varepsilon} \varphi \delta_{\varepsilon}-\int_{\mathbf{R}^{3}} \varphi^{2} \delta_{\varepsilon}
$$

which implies

$$
\liminf _{\varepsilon \rightarrow 0} \int_{\mathbf{R}^{3}}\left|\Phi_{\varepsilon}\right|^{2} \delta_{\varepsilon} \geq 2\langle\Phi, \varphi\rangle-\int_{\mathbf{R}^{3}} \varphi^{2} \delta,
$$

where $\langle.,$.$\rangle means the duality product between measures and functions, from which we$ deduce that $: \sup \left\{\langle\Phi, \varphi\rangle \mid \varphi \in C_{c}^{o}\left(\mathbf{R}^{3}\right),\|\varphi\|_{L^{2}(\Omega)} \leq 1\right\}<+\infty$. Riesz's representation 
theorem implies the existence of some $\chi$ in $L_{\delta}^{2}(\Omega)$ such that for every $\varphi$ in $C_{c}^{o}\left(\mathbf{R}^{3}\right)$ : $\langle\Phi, \varphi\rangle=\int_{\mathbf{R}^{3}} \chi \varphi \delta=\int_{\Omega} \chi \varphi d x$. For every $\varphi$ in $C_{0}^{1}(\Omega)$, one has

$$
\begin{aligned}
\lim _{\varepsilon \rightarrow 0} \frac{|\Omega|}{\left|T_{\varepsilon}\right|} \int_{T_{\varepsilon}} e_{33}\left(u_{\varepsilon}\right) \varphi d x & =\int_{\Omega} \chi \varphi d x \\
& =-\lim _{\varepsilon \rightarrow 0} \frac{|\Omega|}{\left|T_{\varepsilon}\right|} \int_{T_{\varepsilon}} \frac{\partial \varphi}{\partial x_{3}}\left(u_{\varepsilon}\right)_{3} d x \\
& \rightarrow-\int_{\Omega} \frac{\partial \varphi}{\partial x_{3}} v_{3} d x=\int_{\Omega} \varphi e_{33}(v) d x .
\end{aligned}
$$

We thus get : $\int_{\Omega}\left(\chi \varphi-\varphi e_{33}(v)\right) d x=0$, which implies that $e_{33}(v)(=\chi)$ belongs to $L^{2}(\Omega)$.

In order to prove that $v_{i}$ belongs to $L^{2}(\Omega)$, for $i=1,2,3$, we repeat the above argument with $\Phi_{\varepsilon, i}=\left(u_{\varepsilon}\right)_{i}$ instead of $\Phi_{\varepsilon}=e_{33}\left(u_{\varepsilon}\right)$ and we use the estimates of Lemma 13 .

In order to prove that $v_{3}$ is equal to 0 on $\Gamma_{1}$, let us take any function $\varphi$ in $C^{1}(\bar{\Omega})$ taking the form: $\varphi(x)=\theta\left(x_{1}, x_{2}\right) \psi\left(x_{3}\right)$, with $\psi(0)=1, \psi(L)=0, \theta$ in $C^{\infty}(\omega)$. We first compute

$$
\begin{aligned}
\int_{\Omega} \frac{\partial v_{3}}{\partial x_{3}} \varphi d x & \\
\quad= & -\int_{\Omega} \frac{\partial \varphi}{\partial x_{3}} v_{3} d x+\lim _{\varepsilon \rightarrow 0} \frac{|\Omega|}{\left|T_{\varepsilon}\right|} \int_{T_{\varepsilon}}\left(\begin{array}{c}
\left(\varphi\left(u_{\varepsilon}\right)_{3}\right)\left(x_{1}, x_{2}, L\right) \\
-\left(\varphi\left(u_{\varepsilon}\right)_{3}\right)\left(x_{1}, x_{2}, 0\right)
\end{array}\right) d x_{1} d x_{2} \\
\quad= & -\int_{\Omega} \frac{\partial \varphi}{\partial x_{3}} v_{3} d x
\end{aligned}
$$

thanks to the boundary conditions verified by $\varphi$ and $u_{\varepsilon}$. Moreover, using Green's formula, we get

$$
\int_{\Omega} \frac{\partial v_{3}}{\partial x_{3}} \varphi d x=-\int_{\Omega} \frac{\partial \varphi}{\partial x_{3}} v_{3} d x+\int_{\omega} \theta\left(x_{1}, x_{2}\right) v_{3}\left(x_{1}, x_{2}, 0\right) d x_{1} d x_{2},
$$

which implies

$$
\int_{\omega} \theta\left(x_{1}, x_{2}\right) v_{3}\left(x_{1}, x_{2}, 0\right) d x_{1} d x_{2}=0 \Rightarrow v_{3}\left(x_{1}, x_{2}, 0\right)=0
$$

Thus $v$ belongs to $V$.

In order to prove this second assertion, we write the subdifferential inequality for the first term of $F^{\varepsilon}\left(u_{\varepsilon}\right)$

$$
\begin{aligned}
\int_{\Omega \backslash \overline{C_{\varepsilon} \cup T_{\varepsilon}}} \sigma_{i j}\left(u_{\varepsilon}\right) e_{i j}\left(u_{\varepsilon}\right) d x \geq & \int_{\Omega \backslash \overline{C_{\varepsilon} \cup T_{\varepsilon}}} \sigma_{i j}\left(\left(u^{n}\right)_{\varepsilon}^{o}\right) e_{i j}\left(\left(u^{n}\right)_{\varepsilon}^{o}\right) d x \\
& +2 \int_{\Omega \backslash \overline{C_{\varepsilon} \cup T_{\varepsilon}}} \sigma_{i j}\left(u^{n}\right) e_{i j}\left(u_{\varepsilon}-\left(u^{n}\right)_{\varepsilon}^{o}\right) d x,
\end{aligned}
$$

where $\left(u^{n}\right)_{\varepsilon}^{o}$ is associated to $u^{n}$ through (16). The sequence $\left(\left(u^{n}\right)_{\varepsilon}^{o}\right)_{\varepsilon}$ converges to $u^{n}$ in the weak topology of $H^{1}\left(\Omega, \mathbf{R}^{3}\right)$, thanks to Lemma 9, and coincides with $u_{n}$ in $\Omega \backslash \overline{C_{\varepsilon} \cup T_{\varepsilon}}$. 
Thus, $\left(e_{i j}\left(u_{\varepsilon}-\left(u^{n}\right)_{\varepsilon}^{o}\right)\right)_{\varepsilon}$ converges to $e_{i j}\left(u-u^{n}\right)$ in the weak topology of $L^{2}(\Omega)$, for $i, j=1,2,3$. The sequence of characteristic functions of $\Omega \backslash \overline{C_{\varepsilon} \cup T_{\varepsilon}}$ converges to 1 in the strong topology of $L^{2}(\Omega)$. This implies the following convergence

$$
\begin{aligned}
\liminf _{\varepsilon \rightarrow 0} \int_{\Omega \backslash \overline{C_{\varepsilon} \cup T_{\varepsilon}}} \sigma_{i j}\left(u_{\varepsilon}\right) e_{i j}\left(u_{\varepsilon}\right) d x & \geq \int_{\Omega} \sigma_{i j}\left(u^{n}\right) e_{i j}\left(u^{n}\right) d x \\
& +2 \int_{\Omega} \sigma_{i j}\left(u^{n}\right) e_{i j}\left(u-u^{n}\right) d x .
\end{aligned}
$$

Letting $n$ increase to $+\infty$ we get, using the convergence of $\left(u^{n}\right)_{n}$ to $u$ in the strong topology of $H^{1}\left(\Omega, \mathbf{R}^{3}\right)$

$$
\liminf _{\varepsilon \rightarrow 0} \int_{\Omega \backslash \bar{C}_{\varepsilon} \cup T_{\varepsilon}} \sigma_{i j}\left(u_{\varepsilon}\right) e_{i j}\left(u_{\varepsilon}\right) d x \geq \int_{\Omega} \sigma_{i j}(u) e_{i j}(u) d x .
$$

We then write the subdifferential inequality for the second term of $F^{\varepsilon}\left(u_{\varepsilon}\right)$

$$
\begin{aligned}
\int_{C_{\varepsilon}} \sigma_{i j}\left(u_{\varepsilon}\right) e_{i j}\left(u_{\varepsilon}\right) d x & \geq \int_{C_{\varepsilon}} \sigma_{i j}\left(\left(u^{n}\right)_{\varepsilon}^{o}\right) e_{i j}\left(\left(u^{n}\right)_{\varepsilon}^{o}\right) d x \\
+ & 2 \int_{C_{\varepsilon}} \sigma_{i j}\left(\left(u^{n}\right)_{\varepsilon}^{o}\right) e_{i j}\left(u_{\varepsilon}-\left(u_{\varepsilon}^{n}\right)_{\varepsilon}^{o}\right) d x,
\end{aligned}
$$

with

$$
\begin{aligned}
2 \int_{C_{\varepsilon}} \sigma_{i j}\left(\left(u^{n}\right)_{\varepsilon}^{o}\right) & e_{i j}\left(u_{\varepsilon}-\left(u^{n}\right)_{\varepsilon}^{o}\right) d x=2 \int_{C_{\varepsilon}} \sigma_{i j}\left(u^{n}\right) e_{i j}\left(u_{\varepsilon}-\left(u^{n}\right)_{\varepsilon}^{o}\right) d x \\
& +2 \int_{C_{\varepsilon}} \sigma_{i j}\left(z_{\varepsilon}^{m}\left(\left(\mathcal{R}_{\varepsilon}\left(v^{n}\right)\right)_{m}-\left(u^{n}\right)_{m}\right)\right) e_{i j}\left(u_{\varepsilon}-\left(u^{n}\right)_{\varepsilon}^{o}\right) d x .
\end{aligned}
$$

We immediately get : $\lim _{\varepsilon \rightarrow 0} \int_{C_{\varepsilon}} \sigma_{i j}\left(u^{n}\right) e_{i j}\left(u_{\varepsilon}-\left(u^{n}\right)_{\varepsilon}^{o}\right) d x=0$, because the sequence $\left(e_{i j}\left(u_{\varepsilon}-\left(u^{n}\right)_{\varepsilon}^{o}\right)\right)_{\varepsilon}$ converges to $e_{i j}\left(u-u^{n}\right)$ in the weak topology of $L^{2}(\Omega)$, for $i, j=1,2,3$ and the sequence of characteristic functions of $C_{\varepsilon}$ converges to 0 in the strong topology of $L^{2}(\Omega)$. The second term of the last equality can be computed as

$$
\begin{aligned}
\int_{C_{\varepsilon}} \sigma_{i j}\left(z_{\varepsilon}^{m}\left(\left(\mathcal{R}_{\varepsilon}\left(v^{n}\right)\right)_{m}-\left(u^{n}\right)_{m}\right)\right) e_{i j}\left(u_{\varepsilon}-\left(u^{n}\right)_{\varepsilon}^{o}\right) d x \\
=\int_{C_{\varepsilon}} \sigma_{i j}\left(z_{\varepsilon}^{m}\right)\left(\left(\mathcal{R}_{\varepsilon}\left(v^{n}\right)\right)_{m}-\left(u^{n}\right)_{m}\right) e_{i j}\left(u_{\varepsilon}-\left(u^{n}\right)_{\varepsilon}^{o}\right) d x \\
\quad+\int_{C_{\varepsilon}} a_{i j s t}\left(z_{\varepsilon}^{m}\right)_{s} \frac{\partial\left(\left(\mathcal{R}_{\varepsilon}\left(v^{n}\right)\right)_{m}-\left(u^{n}\right)_{m}\right)}{\partial x_{t}} e_{i j}\left(u_{\varepsilon}-\left(u^{n}\right)_{\varepsilon}^{o}\right) d x
\end{aligned}
$$

writing : $\sigma_{i j}=a_{i j s t} e_{s t}$. We observe that

$$
\lim _{\varepsilon \rightarrow 0} \int_{C_{\varepsilon}} a_{i j s t}\left(z_{\varepsilon}^{m}\right)_{s} \frac{\partial\left(\left(\mathcal{R}_{\varepsilon}\left(v^{n}\right)\right)_{m}-\left(u^{n}\right)_{m}\right)}{\partial x_{t}} e_{i j}\left(u_{\varepsilon}-\left(u^{n}\right)_{\varepsilon}^{o}\right) d x=0,
$$


because $\left(z_{\varepsilon}^{m}\right)_{\varepsilon}$ converges to 0 in the strong topology of $L^{2}\left(\Omega, \mathbf{R}^{3}\right),\left|\nabla\left(\mathcal{R}_{\varepsilon}\left(v^{n}\right)-u^{n}\right)\right| \leq C_{n}$, in $C_{\varepsilon}$, and $\left(e_{i j}\left(u_{\varepsilon}-\left(u^{n}\right)_{\varepsilon}^{o}\right)\right)_{\varepsilon}$ converges to $e_{i j}\left(u-u^{n}\right)$ in the weak topology of $L^{2}(\Omega)$, for $i, j=1,2,3$. Then, we compute, using the definition of $z_{\varepsilon}^{m}$

$$
\begin{aligned}
\int_{C_{\varepsilon}} \sigma_{i j}\left(z_{\varepsilon}^{m}\right)\left(\left(\mathcal{R}_{\varepsilon}\left(v^{n}\right)\right)_{m}-\left(u^{n}\right)_{m}\right) e_{i j}\left(u_{\varepsilon}-\left(u^{n}\right)_{\varepsilon}^{o}\right) d x \\
=-\sum_{k} \int_{C_{\varepsilon}^{k}} \sigma_{i j}\left(w_{\varepsilon}^{m k}\right) e_{i j}\left(u_{\varepsilon}-\left(u^{n}\right)_{\varepsilon}^{o}\right)\left(\left(\mathcal{R}_{\varepsilon}\left(v^{n}\right)\right)_{m}-\left(u^{n}\right)_{m}\right) \varphi_{\varepsilon}^{k} d x \\
\quad+\sum_{k} \int_{C_{\varepsilon}^{k}} a_{i j s t}\left(\left(\mathcal{R}_{\varepsilon}\left(v^{n}\right)\right)_{m}-\left(u^{n}\right)_{m}\right)\left(e_{m}-w_{\varepsilon}^{m k}\right)_{s} \frac{\partial \varphi_{\varepsilon}^{k}}{\partial x_{t}} e_{i j}\left(u_{\varepsilon}-\left(u^{n}\right)_{\varepsilon}^{o}\right) d x .
\end{aligned}
$$

But, for every $k$, one has, thanks to the definition (12) of $\varphi_{\varepsilon}^{k}$ and using Lemmas 4 and 9 assertion 1.

$$
\begin{aligned}
\mid \int_{C_{\varepsilon}^{k}}\left(\left(\mathcal{R}_{\varepsilon}\left(v^{n}\right)\right)_{m}\right. & \left.-\left(u^{n}\right)_{m}\right)\left(e_{m}-w_{\varepsilon}^{m k}\right)_{s} \frac{\partial \varphi_{\varepsilon}^{k}}{\partial x_{t}} e_{i j}\left(u_{\varepsilon}-\left(u^{n}\right)_{\varepsilon}^{o}\right) d x \mid \\
& \leq \frac{C_{n}\left|\ln s_{\varepsilon}\right|}{\varepsilon^{2}\left|\ln r_{\varepsilon}\right|} \int_{C_{\varepsilon}^{k} \cap\left\{s_{\varepsilon} / 2<R_{\varepsilon}^{k}<s_{\varepsilon}\right\}} R_{\varepsilon}^{k}\left|\nabla\left(u_{\varepsilon}-\left(u^{n}\right)_{\varepsilon}^{o}\right)\right| d x .
\end{aligned}
$$

This implies, because $\left(u_{\varepsilon}\right)_{\varepsilon}$ and $\left(\left(u^{n}\right)_{\varepsilon}^{o}\right)_{\varepsilon}$ are bounded in $H^{1}\left(\Omega, \mathbf{R}^{3}\right)$

$$
\begin{array}{r}
\limsup _{\varepsilon \rightarrow 0}\left|\sum_{k} \int_{C_{\varepsilon}^{k}} a_{i j s t}\left(\left(\mathcal{R}_{\varepsilon}\left(v^{n}\right)\right)_{m}-\left(u^{n}\right)_{m}\right)\left(e_{m}-w_{\varepsilon}^{m k}\right)_{s} \frac{\partial \varphi_{\varepsilon}^{k}}{\partial x_{t}} e_{i j}\left(u_{\varepsilon}-\left(u^{n}\right)_{\varepsilon}^{o}\right) d x\right| \\
\leq \limsup _{\varepsilon \rightarrow 0} \frac{C_{n}\left|\ln s_{\varepsilon}\right|}{\varepsilon\left|\ln r_{\varepsilon}\right|}\left(\int_{\Omega}\left|\nabla\left(u_{\varepsilon}-\left(u^{n}\right)_{\varepsilon}^{o}\right)\right|^{2} d x\right)^{1 / 2}=0,
\end{array}
$$

because $\gamma$ is finite and using the properties of $s_{\varepsilon}$. Similarly, we estimate, using Lemma 4

$$
\begin{aligned}
\left|\sum_{k} \int_{C_{\varepsilon}^{k}} \sigma_{i j}\left(w_{\varepsilon}^{m k}\right) e_{i j}\left(u_{\varepsilon}-\left(u^{n}\right)_{\varepsilon}^{o}\right)\left(\left(\mathcal{R}_{\varepsilon}\left(v^{n}\right)\right)_{m}-\left(v^{n}\right)_{m}\right) \varphi_{\varepsilon}^{k} d x\right| \\
\leq \frac{C_{n} \sqrt{s_{\varepsilon}}}{\left|\ln \left(r_{\varepsilon}\right)\right|}\left(\int_{\Omega}\left|\nabla\left(u_{\varepsilon}-\left(u^{n}\right)_{\varepsilon}^{o}\right)\right|^{2} d x\right)^{1 / 2} \underset{\varepsilon \rightarrow 0}{\rightarrow} 0
\end{aligned}
$$

because $\gamma$ is finite. We then have to compute the limit of the remaining term

$$
\begin{aligned}
& \sum_{k} \int_{C_{\varepsilon}^{k}} \sigma_{i j}\left(w_{\varepsilon}^{m k}\right) e_{i j}\left(u_{\varepsilon}-\left(u^{n}\right)_{\varepsilon}^{o}\right)\left(\left(v^{n}\right)_{m}-\left(u^{n}\right)_{m}\right) \varphi_{\varepsilon}^{k} d x \\
&=-\sum_{k} \int_{C_{\varepsilon}^{k}} \sigma_{i j, j}\left(w_{\varepsilon}^{m k}\right)\left(u_{\varepsilon}-\left(u^{n}\right)_{\varepsilon}^{o}\right)_{i}\left(\left(v^{n}\right)_{m}-\left(u^{n}\right)_{m}\right) \varphi_{\varepsilon}^{k} d x \\
&-\sum_{k} \int_{C_{\varepsilon}^{k}} \sigma_{i j}\left(w_{\varepsilon}^{m k}\right)\left(u_{\varepsilon}-\left(u^{n}\right)_{\varepsilon}^{o}\right)_{i} \frac{\partial\left(\left(\left(v^{n}\right)_{m}-\left(u^{n}\right)_{m}\right) \varphi_{\varepsilon}^{k}\right)}{\partial x_{j}} d x \\
&+\sum_{k} \int_{\partial T_{\varepsilon}^{k}} \sigma_{i j}\left(w_{\varepsilon}^{m k}\right) n_{j}\left(u_{\varepsilon}-\left(u^{n}\right)_{\varepsilon}^{o}\right)_{i}\left(\left(v^{n}\right)_{m}-\left(u^{n}\right)_{m}\right) d x
\end{aligned}
$$


Using the estimates of Lemma 4, we prove that the second term above converges to 0 . Using the properties of $w_{\varepsilon}^{m k}$, the first term above is equal to 0 . Then, the properties of $w_{\varepsilon}^{m k}$ and the convergence of $\left(u_{\varepsilon}-\left(u^{n}\right)_{\varepsilon}^{o}\right)_{\varepsilon}$ to $u-u^{n}$ in the weak topology of $H^{1}\left(\Omega, \mathbf{R}^{3}\right)$ imply

$$
\begin{aligned}
\lim _{\varepsilon \rightarrow 0} \sum_{k} \int_{C_{\varepsilon}^{k}} \sigma_{i j}\left(w_{\varepsilon}^{m k}\right) e_{i j}\left(u_{\varepsilon}\right. & \left.-\left(u^{n}\right)_{\varepsilon}^{o}\right)\left(\left(v^{n}\right)_{m}-\left(u^{n}\right)_{m}\right) \varphi_{\varepsilon}^{k} d x \\
& =2 \pi \gamma \int_{\Omega}\left(v^{n}-u^{n}\right)^{t} A\left(u-u^{n}\right) d x .
\end{aligned}
$$

We let $n$ increase to $+\infty$ and get

$$
\liminf _{\varepsilon \rightarrow 0} \sum_{k} \int_{C_{\varepsilon}^{k}} \sigma_{i j}\left(w_{\varepsilon}^{m k}\right) e_{i j}\left(u_{\varepsilon}-\left(u^{n}\right)_{\varepsilon}^{o}\right)\left(\left(v^{n}\right)_{m}-\left(u^{n}\right)_{m}\right) \varphi_{\varepsilon}^{k} d x \geq 0
$$

which implies, using the computations of the first assertion

$$
\liminf _{\varepsilon \rightarrow 0} \int_{C_{\varepsilon}} \sigma_{i j}\left(u_{\varepsilon}\right) e_{i j}\left(u_{\varepsilon}\right) d x \geq 2 \pi \gamma \int_{\Omega}(v-u)^{t} A(v-u) d x .
$$

We finally observe that for the third term of $F^{\varepsilon}\left(u_{\varepsilon}\right)$, one has

$$
\int_{T_{\varepsilon}} \sigma_{i j}^{\varepsilon}\left(u_{\varepsilon}\right) e_{i j}\left(u_{\varepsilon}\right) d x \geq \mu^{\varepsilon} \frac{2 \mu^{\varepsilon}+3 \lambda^{\varepsilon}}{\mu^{\varepsilon}+\lambda^{\varepsilon}} \int_{T_{\varepsilon}}\left(e_{33}\left(u_{\varepsilon}\right)\right)^{2} d x .
$$

Indeed, one can easily verify that for every $x, y, z$ in $\mathbf{R}$, one has

$$
\lambda^{\varepsilon}(x+y+z)^{2}+2 \mu^{\varepsilon}\left(x^{2}+y^{2}+z^{2}\right) \geq \mu^{\varepsilon} \frac{2 \mu^{\varepsilon}+3 \lambda^{\varepsilon}}{\mu^{\varepsilon}+\lambda^{\varepsilon}} z^{2} .
$$

We then use the computations given in Lemma 10, which imply, because $\mu_{o}$ and $\lambda_{o}$ are finite

$$
\liminf _{\varepsilon \rightarrow 0} \int_{T_{\varepsilon}} \sigma_{i j}^{\varepsilon}\left(u_{\varepsilon}\right) e_{i j}\left(u_{\varepsilon}\right) d x \geq \pi E_{o} \int_{\Omega}\left(e_{33}(v)\right)^{2} d x .
$$

One deduces from (25)-(27)

$$
\begin{array}{r}
\liminf _{\varepsilon \rightarrow 0} F^{\varepsilon}\left(u_{\varepsilon}\right) \geq \int_{\Omega} \sigma_{i j}(u) e_{i j}(u) d x+2 \pi \gamma \int_{\Omega}(v-u)^{t} A(v-u) d x \\
+\pi E_{o} \int_{\Omega}\left(e_{33}(v)\right)^{2} d x
\end{array}
$$

which concludes the proof.

\subsection{Other situations}

The other situations given by different values of the parameters $\gamma$ or $\lambda_{o}$ or $\mu_{o}$ are summarized in the 
Proposition 11 1. If $\lambda_{o}$ and $\mu_{o}$ are equal to 0 , then $\left(u^{\varepsilon}\right)_{\varepsilon}$ converges in the topology $\tau$ to the solution $\left(u_{o}^{o}, v_{o}^{o}\right)$ of the minimization problem associated to the functional $F_{o}^{o}$ defined in a similar way than (14), but with $\lambda_{o}=\mu_{o}=0$.

2. If $\gamma$ is equal to $+\infty$, one obtains $u^{o \infty}=v^{o \infty}$ in $\Omega$ and $F^{o \infty}$ only depends on $u$

$$
F^{o \infty}(u)=\int_{\Omega} \sigma_{i j}(u) e_{i j}(u) d x+\pi E_{o} \int_{\Omega}\left(e_{33}(u)\right)^{2} d x .
$$

Proof. 1. This case corresponds to a situation where the Lamé coefficients $\lambda^{\varepsilon}$ and $\mu^{\varepsilon}$ of the reinforcing material are smaller than the critical ones given in (10), that is given by

$$
\lambda_{c}^{\varepsilon}=\frac{c \varepsilon^{2}}{\left(r_{\varepsilon}\right)^{2}}, \mu_{c}^{\varepsilon}=\frac{c \varepsilon^{2}}{\left(r_{\varepsilon}\right)^{2}},
$$

for every positive and small $c$, but preserving the critical radius $r_{\varepsilon}$ of the fibers given through $\gamma$. Let $F_{c}^{\varepsilon}$ be the functional defined in (4) but with these critical Lamé coefficients. Thanks to the property of the epi-convergence, we get, for every $(u, v)$ in $H_{\Gamma_{1}}^{1}\left(\Omega, \mathbf{R}^{3}\right) \times V$

$$
\begin{aligned}
F_{o}^{o}(u, v) \leq F_{c}^{o}(u, v)=\int_{\Omega} \sigma_{i j}(u) e_{i j}(u) & d x+\pi c E_{o} \int_{\Omega}\left(e_{33}(v)\right)^{2} d x \\
& +2 \pi \gamma \int_{\Omega}(v-u)^{t} A(v-u) d x .
\end{aligned}
$$

This inequality being true for every positive $c$, we get, letting $c$ go to 0

$$
F_{o}^{o}(u, v) \leq \int_{\Omega} \sigma_{i j}(u) e_{i j}(u) d x+2 \pi \gamma \int_{\Omega}(v-u)^{t} A(v-u) d x .
$$

In order to establish the reverse inequality, we observe that, for every sequence $\left(u_{\varepsilon}\right)_{\varepsilon}$ converging to $(u, v)$ in the above-defined topology $\tau$, one has

$$
F^{\varepsilon}\left(u_{\varepsilon}\right) \geq \int_{\Omega \backslash \overline{B_{\varepsilon}}} \sigma_{i j}\left(u_{\varepsilon}\right) e_{i j}\left(u_{\varepsilon}\right) d x+\int_{C_{\varepsilon}} \sigma_{i j}\left(u_{\varepsilon}\right) e_{i j}\left(u_{\varepsilon}\right) d x,
$$

thus omitting the integral involving the fibers $T_{\varepsilon}$. We then adapt the proof of the second assertion in the Theorem 6 in order to conclude

2. We again observe that this situation corresponds to a case where the Lamé coefficients of the reinforcing material are still given by (10) but where the radius of the fibers is larger than the critical one, that is $r_{\varepsilon} \geq \exp \left(-1 / C \varepsilon^{2}\right)$, for every positive $C$. The functional $F^{\varepsilon}$ is thus larger than the functional $F^{\varepsilon C}$ given by (44), but with the radius $\exp \left(-1 / C \varepsilon^{2}\right)$. The comparison principle implies that for every $(u, v)$ in $H_{\Gamma_{1}}^{1}\left(\Omega, \mathbf{R}^{3}\right) \times V$

$$
\begin{array}{r}
F^{o \infty}(u, v) \geq F^{o C}(u, v)=\int_{\Omega} \sigma_{i j}(u) e_{i j}(u) d x+\pi E_{o} \int_{\Omega}\left(e_{33}(v)\right)^{2} d x \\
+2 \pi \gamma C \int_{\Omega}(v-u)^{t} A(v-u) d x .
\end{array}
$$


Letting $C$ increase to $+\infty$, we observe that $F^{o \infty}(u, v)$ is finite if and only if the integral $\int_{\Omega}(v-u)^{t} A(v-u) d x=0$, which implies : $u=v$, in $\Omega$. The reverse inequality is still obtained adapting the proof of Theorem 6 (first part) but with $v=u$.

Let us now examine the special case when $\lambda_{o}=\mu_{o}=+\infty$. As a special subcase, [6] have considered the case when $\gamma=+\infty$ and

$$
\frac{\lambda^{\varepsilon}\left(r_{\varepsilon}\right)^{4}}{\varepsilon^{2}} \underset{\varepsilon \rightarrow 0}{\rightarrow} \lambda_{1}, \frac{\mu^{\varepsilon}\left(r_{\varepsilon}\right)^{4}}{\varepsilon^{2}} \underset{\varepsilon \rightarrow 0}{\rightarrow} \mu_{1}
$$

with positive and finite $\lambda_{1}$ and $\mu_{1}$. We now adapt their result considering

Proposition 12 Suppose that the above hypothesis (28) holds true and $\gamma$ belongs to ] $0,+\infty]$. Then, the sequence $\left(u^{\varepsilon}\right)_{\varepsilon}$ converges in the topology $\tau$, to the solution $\left(u^{1}, v^{1}\right)$ of

$$
\min _{H_{\Gamma_{1}}^{1}\left(\Omega, \mathbf{R}^{3}\right) \times V^{\prime}}\left(\begin{array}{r}
\int_{\Omega} \sigma_{i j}(u) e_{i j}(u) d x+2 \pi \gamma \int_{\Omega}(v-u)^{t} A(v-u) d x \\
+\frac{\pi E_{1}}{4} \int_{\Omega}\left(\left(\frac{\partial^{2} v_{1}}{\partial x_{3}^{2}}\right)^{2}+\left(\frac{\partial^{2} v_{2}}{\partial x_{3}^{2}}\right)^{2}\right) d x
\end{array}\right),
$$

with $E_{1}=\mu_{1}\left(3 \lambda_{1}+2 \mu_{1}\right) /\left(\lambda_{1}+\mu_{1}\right)$ and

$$
V^{\prime}=\left\{v_{\alpha} \in L^{2}\left(\omega, H^{2}(0, L)\right) \mid v_{\mid \Gamma_{1}}=0, v_{3}=0\right\} .
$$

Proof. We proceed in a similar way to [6]. Indeed, we first follow their method in order to prove the following estimates

$$
\frac{1}{\left|T_{\varepsilon}\right|} \int_{T_{\varepsilon}}\left|u^{\varepsilon}\right| d x<C, \frac{1}{\left|T_{\varepsilon}\right|} \int_{T_{\varepsilon}}\left|u^{\varepsilon}\right|^{2} d x<C, \frac{1}{\left(r_{\varepsilon}\right)^{2}\left|T_{\varepsilon}\right|} \int_{T_{\varepsilon}}\left|e_{i j}\left(u^{\varepsilon}\right)\right|^{2} d x<C,
$$

where $C$ is independant of $\varepsilon$. For every smooth $v$ in $C^{2}\left(\bar{\Omega}, \mathbf{R}^{3}\right) \cap V^{\prime}$, we set

$$
\left\{\begin{array}{c}
\left(\mathcal{R}_{\varepsilon 1}(v)\right)_{1}\left(x_{1}, x_{2}, x_{3}\right)=v_{1}\left(k_{1} \varepsilon, k_{2} \varepsilon, x_{3}\right) \\
-\frac{\lambda^{\varepsilon}}{2\left(\mu^{\varepsilon}+\lambda^{\varepsilon}\right)} \frac{\left(x_{1}-k_{1} \varepsilon\right)^{2}-\left(x_{2}-k_{2} \varepsilon\right)^{2}}{2} \frac{\partial^{2} v_{1}}{\partial x_{3}^{2}}\left(k_{1} \varepsilon, k_{2} \varepsilon, x_{3}\right) \\
-\frac{\lambda^{\varepsilon}}{2\left(\mu^{\varepsilon}+\lambda^{\varepsilon}\right)} \frac{\left(x_{1}-k_{1} \varepsilon\right)\left(x_{2}-k_{2} \varepsilon\right)}{2} \frac{\partial^{2} v_{2}}{\partial x_{3}^{2}}\left(k_{1} \varepsilon, k_{2} \varepsilon, x_{3}\right) \\
\left(\mathcal{R}_{\varepsilon 1}(v)\right)_{2}\left(x_{1}, x_{2}, x_{3}\right)=v_{2}\left(k_{1} \varepsilon, k_{2} \varepsilon, x_{3}\right) \\
-\frac{\lambda^{\varepsilon}}{2\left(\mu^{\varepsilon}+\lambda^{\varepsilon}\right)} \frac{\left(x_{1}-k_{1} \varepsilon\right)^{2}-\left(x_{2}-k_{2} \varepsilon\right)^{2}}{2} \frac{\partial^{2} v_{2}}{\partial x_{3}^{2}}\left(k_{1} \varepsilon, k_{2} \varepsilon, x_{3}\right) \\
-\frac{\lambda^{\varepsilon}}{2\left(\mu^{\varepsilon}+\lambda^{\varepsilon}\right)} \frac{\left(x_{1}-k_{1} \varepsilon\right)\left(x_{2}-k_{2} \varepsilon\right)}{2} \frac{\partial^{2} v_{1}}{\partial x_{3}^{2}}\left(k_{1} \varepsilon, k_{2} \varepsilon, x_{3}\right) \\
\left(\mathcal{R}_{\varepsilon 1}(v)\right)_{3}\left(x_{1}, x_{2}, x_{3}\right)=-\left(x_{1}-k_{1} \varepsilon\right) \frac{\partial v_{1}}{\partial x_{3}}\left(k_{1} \varepsilon, k_{2} \varepsilon, x_{3}\right) \\
-\left(x_{2}-k_{2} \varepsilon\right) \frac{\partial v_{2}}{\partial x_{3}}\left(k_{1} \varepsilon, k_{2} \varepsilon, x_{3}\right) .
\end{array}\right.
$$


The verification of the first assertion of the epi-convergence is obtained computing the energy of the test-function associated to this $\mathcal{R}_{\varepsilon 1}(v)$. The verification of the second assertion follows the same lines as in Theorem 6 .

Remark 13 The extra term occuring in the energy functional described in Proposition 12 corresponds to the flexion of the fibers.

Remark 14 In the case $\gamma=0$, one can still prove that $\left(\int_{T_{\varepsilon}}\left|\left(u_{\varepsilon}\right)_{3}\right| d x /\left|T_{\varepsilon}\right|\right)$ is bounded, writing : $u_{\varepsilon}(s)=\int_{0}^{s} \partial\left(u_{\varepsilon}\right)_{3} / \partial x_{3} d t$ and using some trivial arguments. Thus Lemma 10 still implies the existence of $e_{33}(v)$ in $L^{2}(\Omega)$, with $v_{3}=0$ on $\Gamma_{1}$. We conjecture that the limit functional is

$$
F^{o o}(u, v)=\int_{\Omega} \sigma_{i j}(u) e_{i j}(u) d x+\pi E_{o} \int_{\Omega}\left(e_{33}(v)\right)^{2} d x .
$$

\section{Further extensions}

\subsection{The case of a almost non-periodic distribution of fibers}

Let $\widetilde{\omega}$ be some open subset of $\mathbf{R}^{2}$ and $\theta$ be a $C^{1}$-diffeomorphism from $\widetilde{\omega}$ to $\omega$. We define the following almost non-periodic distribution of non-homogeneous fibers as follows. The fibers are defined as

$$
T_{\varepsilon}^{k}=\left\{\left(x_{1}, x_{2}, x_{3}\right) \mid\left(x_{1}-\theta_{1}\left(k_{1} \varepsilon, k_{2} \varepsilon\right)\right)^{2}+\left(x_{2}-\theta_{2}\left(k_{1} \varepsilon, k_{2} \varepsilon\right)\right)^{2}<\left(r_{\varepsilon}\right)^{2}, x_{3} \in\right] 0, L[\} .
$$

Replacing $\left(k_{1} \varepsilon, k_{2} \varepsilon\right)$ by $\theta\left(k_{1} \varepsilon, k_{2} \varepsilon\right)$ in the local test-functions and adapting the proof of Theorem 6, one can prove

Theorem 15 Suppose that $\gamma$ is positive and finite and the nonhomogeneous material filling in the fibers satisfies the usual conditions of symmetry, uniform ellipticity and continuity and

$$
\sup _{x_{3} \in[0, L], \varepsilon>0}\left|\frac{\left(r_{\varepsilon}\right)^{2}}{\varepsilon^{2}} a_{i j k l}^{\varepsilon}\left(x_{3}\right)\right|<+\infty, \frac{\left(r_{\varepsilon}\right)^{2}}{\varepsilon^{2}} a_{i j k l}^{\varepsilon}\left(x_{3}\right) \underset{\varepsilon \rightarrow 0}{\rightarrow} a_{i j k l}^{o}\left(x_{3}\right) \text {, a.e. in } \Omega .
$$

Then, the sequence $\left(F^{\varepsilon}\right)_{\varepsilon}$ epi-converges in the topology $\tau$ to the functional $F^{o}$ defined on $H^{1}\left(\Omega, \mathbf{R}^{3}\right) \times L^{1}\left(\Omega, \mathbf{R}^{3}\right)$ by:

$$
F^{o}(u, v)=\left\{\begin{array}{r}
\int_{\Omega} \sigma_{i j}(u) e_{i j}(u) d x+2 \pi \gamma \int_{\Omega}(v-u)^{t} A(v-u)\left|\nabla \theta^{-1}\right|\left(x_{1}, x_{2}\right) d x \\
+\pi \int_{\Omega} E^{o}\left(x_{3}\right) e_{33}(v) e_{33}(v)\left|\nabla \theta^{-1}\right|\left(x_{1}, x_{2}\right) d x \\
\quad \text { if }(u, v) \in H_{\Gamma_{1}}^{1}\left(\Omega, \mathbf{R}^{3}\right) \times V \\
\text { otherwise }
\end{array}\right.
$$

where $E^{o}\left(x_{3}\right)$ is Young's modulus associated to $a_{i j k l}^{o}\left(x_{3}\right)$. 


\subsection{The case of tranverse fibers}

Let us assume in this paragraph that $\omega$ is the disk centred at the origin and of radius $R>0$ of $\mathbf{R}^{2}$. Choose any $R^{*}$ in $\left.] 0, R\right]$ and positive $\varepsilon$ and $r_{\varepsilon}$ such that : $0<2 r_{\varepsilon}<\varepsilon<1$. For every $k$ in $\mathbf{Z}$, we introduce the torus $T_{\varepsilon}^{k}$ defined as

$$
T_{\varepsilon}^{k}=\left\{\left(x_{1}, x_{2}, x_{3}\right) \in \mathbf{R}^{3} \mid\left(R^{*}-\sqrt{\left(x_{1}\right)^{2}+\left(x_{2}\right)^{2}}\right)^{2}+\left(x_{3}-k \varepsilon\right)^{2}<\left(r_{\varepsilon}\right)^{2}\right\} .
$$

$T_{\varepsilon}$ denotes the union $\bigcup_{k=-n(\varepsilon)}^{k=n(\varepsilon)} T_{\varepsilon}^{k}$ of the tori $T_{\varepsilon}^{k} \varepsilon$-periodically distributed along the surface : $\Sigma_{R^{*}}=\left\{\left(x_{1}\right)^{2}+\left(x_{2}\right)^{2}=\left(R^{*}\right)^{2}, x_{3} \in\right] 0, L[\}$ and contained in $\left.\Omega=\omega \times\right] 0, L[$. We suppose that $\overline{T_{\varepsilon}} \cap \Gamma_{1}$ and $\overline{T_{\varepsilon}} \cap \Gamma_{2}$ are empty. The number $n(\varepsilon)$ of such tori contained in $\Omega$ is equivalent to $L / \varepsilon$.

We define the topology $\tau^{*}$ as

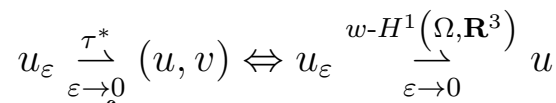

$$
\begin{aligned}
& \text { and : } \forall \varphi \in C_{c}^{o}\left(\mathbf{R}^{3}\right): \int_{\Sigma_{R^{*}}}^{\varepsilon \rightarrow 0}\left(R^{\varepsilon^{*}}\left(u_{\varepsilon}\right) \varphi\right)_{\mid \Sigma_{R^{*}}}^{\varepsilon \rightarrow 0} d \sigma \underset{\varepsilon \rightarrow 0}{\rightarrow} \int_{\Sigma_{R^{*}}}(v \varphi)_{\mid \Sigma_{R^{*}}} d \sigma \text {, }
\end{aligned}
$$

where $R^{\varepsilon^{*}}$ is defined by : $R^{\varepsilon^{*}}(u)=\left|\Sigma_{R^{*}}\right| u \mathbf{1}_{T_{\varepsilon}} /\left|T_{\varepsilon}\right|$. We introduce the space

$$
V^{*}=\left\{\begin{array}{c}
\left.v=\left(v_{r}, v_{\theta}, v_{x_{3}}\right):[0,2 \pi] \times\right] 0, L\left[\rightarrow \mathbf{R}^{3} \mid v_{\alpha} \in L^{2}(] 0,2 \pi[\times] 0, L[),\right. \\
v_{\alpha}(0, .)=v_{\alpha}(2 \pi, .), \alpha=r, \theta, x_{3}, \frac{\partial v_{\theta}}{\partial \theta}+v_{r} \in L^{2}(] 0,2 \pi[\times] 0, L[) .
\end{array}\right\}
$$

Figure 2: The cylinder $\Omega$ and the tori $T_{\varepsilon}^{k}$.

Following similar arguments to the ones presented in the previous parts, we prove 
Theorem 16 Suppose that $\gamma^{*}=\lim _{\varepsilon \rightarrow 0}\left(-1 /\left(\varepsilon \ln r_{\varepsilon}\right)\right)$ is finite, $\lambda_{o}^{*}$ and $\mu_{o}^{*}$ are finite and $\mu_{o}^{*}$ is positive, with:

$$
\lambda_{o}^{*}=\lim _{\varepsilon \rightarrow 0} \frac{\lambda^{\varepsilon}\left(r_{\varepsilon}\right)^{2}}{\varepsilon}, \mu_{o}^{*}=\lim _{\varepsilon \rightarrow 0} \frac{\mu^{\varepsilon}\left(r_{\varepsilon}\right)^{2}}{\varepsilon} .
$$

Then, the sequence $\left(F^{\varepsilon}\right)_{\varepsilon}$ epi-converges in the topology $\tau^{*}$ to the functional $F^{o^{*}}$ defined on $H^{1}\left(\Omega, \mathbf{R}^{3}\right) \times L^{1}\left(\Omega, \mathbf{R}^{3}\right)$ by:

$$
F^{o^{*}}(u, v)=\left\{\begin{array}{r}
\int_{\Omega} \sigma_{i j}(u) e_{i j}(u) d x+\pi E_{o}^{*} \int_{0}^{2 \pi} \int_{0}^{L}\left(\frac{\partial v_{\theta}}{\partial \theta}+v_{r}\right)^{2}\left(R^{*}, \theta, x_{3}\right) d \theta d x_{3} \\
+2 \pi \gamma^{*} R^{*} \int_{0}^{2 \pi} \int_{0}^{L}\left(v-u_{\mid \Sigma_{R^{*}}}\right)^{t} A\left(v-u_{\mid \Sigma_{R^{*}}}\right)\left(R^{*}, \theta, x_{3}\right) d \theta d x_{3} \\
+\infty
\end{array} \quad \begin{array}{r}
\text { if }(u, v) \in H_{\Gamma_{1}}^{1}\left(\Omega, \mathbf{R}^{3}\right) \times V^{*} \\
\text { otherwise }
\end{array}\right.
$$

with $A$ as in Theorem [6 and $E_{o}^{*}=\mu_{o}^{*}\left(3 \lambda_{o}^{*}+2 \mu_{o}^{*}\right) /\left(\lambda_{o}^{*}+\mu_{o}^{*}\right)$.

\section{ACKNOWLEDGEMENT}

We thank the referee for the useful comments on this work.

\section{References}

[1] ATTOUCH, H. 1984 Variational convergence for functions and operators. Appl. Math. Series. London, Pitman.

[2] BELliEUD, M. \& BOUCHITTE, G. 1998 Homogenization of elliptic problems in a fiber reinforced structure. Non-local effects. Ann. Scuola Norm. Sup. Pisa Cl. Sci. (4) 26, 407-436.

[3] CAILlERIE, D. \& DINARI, B. 1987 A perturbation problem with two small parameters in the framework of the heat conduction of a fiber reinforced body. Partial differential equations. Warsaw, Banach Center Publications.

[4] KHRUSLOV, E. Ya. 1991 Homogenization models of composite media. Composite media and homogenization. G. Dal Maso and G. Dell'Antonio Eds. Basel, Birkhaüser.

[5] MOSCO, U. 1994 Composite media and Dirichlet forms. J. Funct. Anal. 123, 368-421.

[6] PIDERI, C. \& SEPPECHER, P. 1997 A second gradient material resulting from the homogenization of an heterogeneous linear elastic medium. Continuum Mech. Thermo. 9, 241-257.

[7] SOKOLNIKOFF, I. S. 1956 Mathematical theory of elasticity. New York, Mc Graw Hill. 